\title{
Weak Convergence Rates for Spatial Spectral Galerkin Approximations of Semilinear Stochastic Wave Equations with Multiplicative Noise
}

\author{
Ladislas Jacobe de Naurois $^{1} \cdot$ Arnulf Jentzen ${ }^{2,3} \cdot$ Timo Welti $^{2,4}{ }_{\mathbb{C}}$
}

Accepted: 29 December 2020 / Published online: 6 November 2021

(c) The Author(s) 2021

\begin{abstract}
Stochastic wave equations appear in several models for evolutionary processes subject to random forces, such as the motion of a strand of DNA in a liquid or heat flow around a ring. Semilinear stochastic wave equations can typically not be solved explicitly, but the literature contains a number of results which show that numerical approximation processes converge with suitable rates of convergence to solutions of such equations. In the case of approximation results for strong convergence rates, semilinear stochastic wave equations with both additive or multiplicative noise have been considered in the literature. In contrast, the existing approximation results for weak convergence rates assume that the diffusion coefficient of the considered semilinear stochastic wave equation is constant, that is, it is assumed that the considered wave equation is driven by additive noise, and no approximation results for multiplicative noise are known. The purpose of this work is to close this gap and to establish essentially sharp weak convergence rates for spatial spectral Galerkin approximations of semilinear stochastic wave equations with multiplicative noise. In particular, our weak convergence result establishes as a special case essentially sharp weak convergence rates for the continuous version of the hyperbolic Anderson model. Our method of proof makes use of the Kolmogorov equation and the Hölder-inequality for Schatten norms.
\end{abstract}

$\bowtie \quad$ Timo Welti

contact@twelti.org

Ladislas Jacobe de Naurois

1ldenaurois@gmail.com

Arnulf Jentzen

a.j@uni-muenster.de

1 Zürich, Switzerland

2 Seminar for Applied Mathematics, Department of Mathematics, ETH Zürich, 8092 Zürich, Switzerland

3 Faculty of Mathematics and Computer Science, University of Münster, 48149 Münster, Germany

4 D ONE Solutions AG, 8003 Zürich, Switzerland 
Keywords weak convergence $\cdot$ stochastic wave equations $\cdot$ multiplicative noise . hyperbolic Anderson model $\cdot$ spatial approximation

\section{Introduction}

In the field of numerical approximation of stochastic evolution equations (SEEs) one distinguishes between two conceptually fundamentally different error criteria, that is, strong convergence and weak convergence. In the case of the finite-dimensional stochastic ordinary differential equations, both strong and weak convergence are quite well understood nowadays; cf., e.g., the standard monographs Kloeden \& Platen [26] and Milstein [36]. However, the situation is different in the case of the infinitedimensional stochastic partial differential equations (cf., e.g., Walsh [43], Da Prato \& Zabczyk [13], Liu \& Röckner [35]). In the case of stochastic partial differential equations with regular nonlinearities, strong convergence rates are essentially well understood, whereas a proper understanding of weak convergence rates has still not been reached (cf., e.g., [1,2,4-6,9,11,16-22,25,27-29,31,32,34,41,45-47] for several weak convergence results in the literature). In this work we derive weak convergence rates for stochastic wave equations. Stochastic wave equations can be used for modelling several evolutionary processes subject to random forces. Examples include the motion of a DNA molecule floating in a fluid and the dilatation of shock waves throughout the sun (cf., e.g., Section 1 in Dalang [14]), as well as heat conduction around a ring (cf., e.g., Thomas [42]). Unfortunately, such problems usually involve complicated nonlinearities and are inaccessible for current numerical analysis approaches. Nonetheless, rigorous examination of simpler model problems such as the ones considered in the present work is a key first step. Even though a number of strong convergence rates for stochastic wave equations are available (cf., e.g., [3,7,8,30,39,44,45,48]), apart from the findings of the works Harms \& Müller [20] and Cox et al. [11], which have appeared after the preprint [23] of the present article, the existing weak convergence results for stochastic wave equations in the literature (cf., e.g., [22,28,29,31,45,46]) assume that the diffusion coefficient is constant or, in other words, that the equation is driven by additive noise.

The main contribution of this work is the derivation of essentially sharp weak convergence rates for a class of stochastic wave equations large enough to also include the case of multiplicative noise. Roughly speaking, the main result of this article (cf. Theorem 3.6 in Sect. 3.2 below) establishes upper bounds for weak errors associated to spatial spectral Galerkin approximations of semilinear stochastic wave equations under suitable Lipschitz and smoothness assumptions on the drift nonlinearity and on the diffusion coefficient as well as under suitable integrability and regularity assumptions on the initial value. In order to employ a mild solution framework, the second-order stochastic wave equations are formulated as first-order two-component systems of SEEs on an extended state space. The first component process of the solution process of such a first-order system corresponds to the solution process of the original second-order equation, while the second component process corresponds to the time derivative of the first component process. As is often the case in the context of spatial spectral Galerkin approximations, convergence is obtained in terms of the in absolute value increasing sequence of eigenvalues of a symmetric linear operator. 
To illustrate the main result of this article in more detail, we consider the following setting as a special case of our general framework (cf. Sect. 3.1 below). Consider the notation in Sect. 1.1, let $\left(H,\langle\cdot, \cdot\rangle_{H},\|\cdot\|_{H}\right)$ and $\left(U,\langle\cdot, \cdot\rangle_{U},\|\cdot\|_{U}\right)$ be separable $\mathbb{R}$-Hilbert spaces, let $T \in(0, \infty)$, let $(\Omega, \mathcal{F}, \mathbb{P})$ be a probability space with a normal filtration $\left(\mathbb{F}_{t}\right)_{t \in[0, T]}$, let $\left(W_{t}\right)_{t \in[0, T]}$ be an $\operatorname{id}_{U}$-cylindrical $\left(\Omega, \mathcal{F}, \mathbb{P},\left(\mathbb{F}_{t}\right)_{t \in[0, T]}\right)$-Wiener

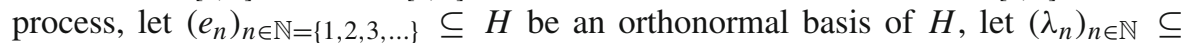
$(0, \infty)$ be an increasing sequence, let $A: D(A) \subseteq H \rightarrow H$ be the linear operator which satisfies $D(A)=\left\{v \in H: \sum_{n=1}^{\infty}\left|\lambda_{n}\left\langle e_{n}, v\right\rangle_{H}\right|^{2}<\infty\right\}$ and $\forall v \in D(A): A v=$ $\sum_{n=1}^{\infty}-\lambda_{n}\left\langle e_{n}, v\right\rangle_{H} e_{n}$, let $\left(H_{r},\langle\cdot, \cdot\rangle_{H_{r}},\|\cdot\|_{H_{r}}\right), r \in \mathbb{R}$, be a family of interpolation spaces associated to $-A$ (cf., e.g., [40, Section 3.7]), let $\left(\mathbf{H}_{r},\langle\cdot, \cdot\rangle_{\mathbf{H}_{r}},\|\cdot\|_{\mathbf{H}_{r}}\right), r \in \mathbb{R}$, be the family of $\mathbb{R}$-Hilbert spaces which satisfies for all $r \in \mathbb{R}$ that $\left(\mathbf{H}_{r},\langle\cdot, \cdot\rangle_{\mathbf{H}_{r}},\|\cdot\|_{\mathbf{H}_{r}}\right)=$ $\left(H_{r / 2} \times H_{r / 2-1 / 2},\langle\cdot, \cdot\rangle_{H_{r / 2} \times H_{r / 2-1 / 2}},\|\cdot\|_{H_{r / 2} \times H_{r / 2-1 / 2}}\right)$, let $\mathbf{P}_{N} \in L\left(\mathbf{H}_{0}\right), N \in \mathbb{N} \cup\{\infty\}$, be the linear operators which satisfy for all $N \in \mathbb{N} \cup\{\infty\}, v, w \in H$ that $\mathbf{P}_{N}(v, w)=$ $\left(\sum_{n=1}^{N}\left\langle e_{n}, v\right\rangle_{H} e_{n}, \sum_{n=1}^{N}\left\langle e_{n}, w\right\rangle_{H} e_{n}\right)$, let $\mathbf{A}: D(\mathbf{A}) \subseteq \mathbf{H}_{0} \rightarrow \mathbf{H}_{0}$ be the linear operator which satisfies $D(\mathbf{A})=\mathbf{H}_{1}$ and $\forall(v, w) \in \mathbf{H}_{1}: \mathbf{A}(v, w)=(w, A v)$, and let $\gamma \in$ $(0, \infty), \beta \in(\gamma / 2, \gamma], \rho \in[0,2(\gamma-\beta)], \varrho, C_{\mathbf{F}}, C_{\mathbf{B}} \in[0, \infty), \xi \in L^{2}\left(\left.\mathbb{P}\right|_{\mathbb{F}_{0}} ; \mathbf{H}_{2(\gamma-\beta)}\right)$, $\mathbf{F} \in \operatorname{Lip}^{0}\left(\mathbf{H}_{0}, \mathbf{H}_{0}\right)$, and $\mathbf{B} \in \operatorname{Lip}^{0}\left(\mathbf{H}_{0}, L_{2}\left(U, \mathbf{H}_{0}\right)\right)$ satisfy $(-A)^{-\beta} \in L_{1}(H)$, $\mathbf{F}\left(\mathbf{H}_{\rho}\right) \subseteq \mathbf{H}_{2(\gamma-\beta)},\left(\mathbf{H}_{\rho} \ni v \mapsto \mathbf{F}(v) \in \mathbf{H}_{2(\gamma-\beta)}\right) \in \operatorname{Lip}^{0}\left(\mathbf{H}_{\rho}, \mathbf{H}_{2(\gamma-\beta)}\right), \forall v \in$ $\mathbf{H}_{\rho}, u \in U: \mathbf{B}(v) u \in \mathbf{H}_{\gamma}, \forall v \in \mathbf{H}_{\rho}:\left(U \ni u \mapsto \mathbf{B}(v) u \in \mathbf{H}_{\rho}\right) \in L_{2}\left(U, \mathbf{H}_{\rho}\right)$, $\left(\mathbf{H}_{\rho} \ni v \mapsto\left(U \ni u \mapsto \mathbf{B}(v) u \in \mathbf{H}_{\rho}\right) \in L_{2}\left(U, \mathbf{H}_{\rho}\right)\right) \in \operatorname{Lip}^{0}\left(\mathbf{H}_{\rho}, L_{2}\left(U, \mathbf{H}_{\rho}\right)\right)$, $\forall v \in \mathbf{H}_{\rho}:\left(U \ni u \mapsto \mathbf{B}(v) u \in \mathbf{H}_{\gamma}\right) \in L\left(U, \mathbf{H}_{\gamma}\right),\left(\mathbf{H}_{\rho} \ni v \mapsto(U \ni u \mapsto \mathbf{B}(v) u \in\right.$ $\left.\left.\mathbf{H}_{\gamma}\right) \in L\left(U, \mathbf{H}_{\gamma}\right)\right) \in \operatorname{Lip}^{0}\left(\mathbf{H}_{\rho}, L\left(U, \mathbf{H}_{\gamma}\right)\right),\left.\mathbf{F}\right|_{\mathbf{H}_{\varrho}} \in C^{2}\left(\mathbf{H}_{\varrho}, \mathbf{H}_{0}\right),\left.\mathbf{B}\right|_{\mathbf{H}_{\varrho}} \in C^{2}\left(\mathbf{H}_{\varrho}\right.$, $\left.L_{2}\left(U, \mathbf{H}_{0}\right)\right), C_{\mathbf{F}}=\sup _{x, v_{1}, v_{2} \in \cap_{r \in \mathbb{R}} \mathbf{H}_{r}, \max \left\{\left\|v_{1}\right\|_{\mathbf{H}_{0}},\left\|v_{2}\right\|_{\mathbf{H}_{0}}\right\} \leq 1}\left\|\mathbf{F}^{\prime \prime}(x)\left(v_{1}, v_{2}\right)\right\|_{\mathbf{H}_{0}}<\infty$, and $C_{\mathbf{B}}=\sup _{x, v_{1}, v_{2} \in \cap_{r \in \mathbb{R}} \mathbf{H}_{r}, \max \left\{\left\|v_{1}\right\|_{\mathbf{H}_{0}},\left\|v_{2}\right\|_{\mathbf{H}_{0}}\right\} \leq 1}\left\|\mathbf{B}^{\prime \prime}(x)\left(v_{1}, v_{2}\right)\right\|_{L_{2}\left(U, \mathbf{H}_{0}\right)}<\infty$.

Theorem 1.1 Assume the above setting. Then

(i) it holds that there exist up to modifications unique $\left(\mathbb{F}_{t}\right)_{t \in[0, T] \text {-predictable }}$ stochastic processes $\mathbf{X}^{N}=\left(X^{N}, \mathcal{X}^{N}\right):[0, T] \times \Omega \rightarrow \mathbf{P}_{N}\left(\mathbf{H}_{\rho}\right), N \in \mathbb{N} \cup\{\infty\}$, such that for all $N \in \mathbb{N} \cup\{\infty\}, t \in[0, T]$ it holds that $\sup _{s \in[0, T]} \mathbb{E}\left[\left\|\mathbf{X}_{s}^{N}\right\|_{\mathbf{H}_{\rho}}^{2}\right]<$ $\infty$ and $\mathbb{P}$-a.s. that

$$
\mathbf{X}_{t}^{N}=\mathrm{e}^{t \mathbf{A}} \mathbf{P}_{N} \xi+\int_{0}^{t} \mathrm{e}^{(t-s) \mathbf{A}} \mathbf{P}_{N} \mathbf{F}\left(\mathbf{X}_{s}^{N}\right) \mathrm{d} s+\int_{0}^{t} \mathrm{e}^{(t-s) \mathbf{A}} \mathbf{P}_{N} \mathbf{B}\left(\mathbf{X}_{s}^{N}\right) \mathrm{d} W_{s}
$$

and

(ii) it holds that

$$
\begin{aligned}
& \sup _{N \in \mathbb{N}} \sup _{\varphi \in C_{\mathrm{b}}^{2}\left(\mathbf{H}_{0}, \mathbb{R}\right) \backslash\{0\}}\left(\frac{\left(\lambda_{N}\right)^{\gamma-\beta}\left|\mathbb{E}\left[\varphi\left(\mathbf{X}_{T}^{\infty}\right)\right]-\mathbb{E}\left[\varphi\left(\mathbf{X}_{T}^{N}\right)\right]\right|}{\|\varphi\|_{C_{\mathrm{b}}^{2}\left(\mathbf{H}_{0}, \mathbb{R}\right)}}\right) \\
& \leq\left[\mathbb{E}\left[\|\xi\|_{\mathbf{H}_{2(\gamma-\beta)}}\right]+T\left\|\left.\mathbf{F}\right|_{\mathbf{H}_{\rho}}\right\|_{\operatorname{Lip}^{0}\left(\mathbf{H}_{\rho}, \mathbf{H}_{2(\gamma-\beta)}\right)}\right. \\
& \left.\quad+2 T\left\|(-A)^{-\beta}\right\|_{L_{1}(H)}\left\|\left.\mathbf{B}\right|_{\mathbf{H}_{\rho}}\right\|_{\operatorname{Lip}^{0}\left(\mathbf{H}_{\rho}, L\left(U, \mathbf{H}_{\gamma}\right)\right)}^{2}\right] \\
& \quad \cdot \max \left\{1,\left[T\left(\left(C_{\mathbf{F}}\right)^{2}+2\left(C_{\mathbf{B}}\right)^{2}\right)\right]^{1 / 2}\right\} \\
& \quad \cdot \exp \left(T\left[\frac{1}{2}+3|\mathbf{F}|_{\operatorname{Lip}^{0}\left(\mathbf{H}_{0}, \mathbf{H}_{0}\right)}+4|\mathbf{B}|_{\operatorname{Lip}^{0}\left(\mathbf{H}_{0}, L_{2}\left(U, \mathbf{H}_{0}\right)\right)}^{2}\right]\right)
\end{aligned}
$$




$$
\begin{aligned}
& \cdot \max \left\{1, \mathbb{E}\left[\|\xi\|_{\mathbf{H}_{\rho}}^{2}\right]\right\} \\
& \cdot \exp \left(T\left[2\left\|\left.\mathbf{F}\right|_{\mathbf{H}_{\rho}}\right\|_{\operatorname{Lip}^{0}\left(\mathbf{H}_{\rho}, \mathbf{H}_{\rho}\right)}+\left\|\left.\mathbf{B}\right|_{\mathbf{H}_{\rho}}\right\|_{\operatorname{Lip}^{0}\left(\mathbf{H}_{\rho}, L_{2}\left(U, \mathbf{H}_{\rho}\right)\right)}^{2}\right]\right)<\infty
\end{aligned}
$$

Theorem 1.1 is a consequence of the more general results in Remark 3.1 and Theorem 3.6 below (cf. Corollary 3.8). Let us now add a few remarks regarding Theorem 1.1.

First, we briefly outline our proof of Theorem 1.1. As usual in the case of weak convergence analysis, the Kolmogorov equation (cf. (3.25) below) is used. Another key ingredient is the Hölder inequality for Schatten norms (cf. (3.28) below). In addition, the proof of Theorem 1.1 employs the mild Itô formula (cf. Corollary 1 in Da Prato et al. [12]) to obtain suitable a priori estimates for solutions of (1.1) (cf. Lemma 3.2 in Sect. 3.2 below for details). The detailed proof of Theorems 1.1 and 3.6, respectively, can be found in Sect. 3.2.

Second, we would like to emphasize that in the general setting of Theorem 1.1, the weak convergence rate established in Theorem 1.1 can essentially not be improved. More precisely, Theorem 1.1 in [24] proves that for every $\eta \in(0, \infty)$ and every infinite-dimensional separable $\mathbb{R}$-Hilbert space $\left(H,\langle\cdot, \cdot\rangle_{H},\|\cdot\|_{H}\right)$ there exist $\left(U,\langle\cdot, \cdot\rangle_{U},\|\cdot\|_{U}\right), A: D(A) \subseteq H \rightarrow H, \gamma, c \in(0, \infty),\left(C_{\varepsilon}\right)_{\varepsilon \in(0, \infty)} \subseteq$ $[0, \infty), \rho \in[0, \gamma / 2], \xi \in L^{2}\left(\left.\mathbb{P}\right|_{\mathbb{F}_{0}} ; \mathbf{H}_{\gamma}\right), \varphi \in C_{\mathrm{b}}^{2}\left(\mathbf{H}_{0}, \mathbb{R}\right), \mathbf{F} \in C_{\mathrm{b}}^{2}\left(\mathbf{H}_{0}, \mathbf{H}_{0}\right)$, and $\mathbf{B} \in C_{\mathrm{b}}^{2}\left(\mathbf{H}_{0}, L_{2}\left(U, \mathbf{H}_{0}\right)\right)$ such that $\mathbf{F}\left(\mathbf{H}_{\rho}\right) \subseteq \mathbf{H}_{\gamma},\left(\mathbf{H}_{\rho} \ni v \mapsto \mathbf{F}(v) \in \mathbf{H}_{\gamma}\right) \in$ $\operatorname{Lip}^{0}\left(\mathbf{H}_{\rho}, \mathbf{H}_{\gamma}\right), \forall v \in \mathbf{H}_{\rho}, u \in U: \mathbf{B}(v) u \in \mathbf{H}_{\gamma}, \forall v \in \mathbf{H}_{\rho}:(U \ni u \mapsto \mathbf{B}(v) u \in$ $\left.\mathbf{H}_{\rho}\right) \in L_{2}\left(U, \mathbf{H}_{\rho}\right),\left(\mathbf{H}_{\rho} \ni v \mapsto\left(U \ni u \mapsto \mathbf{B}(v) u \in \mathbf{H}_{\rho}\right) \in L_{2}\left(U, \mathbf{H}_{\rho}\right)\right) \in$ $\operatorname{Lip}^{0}\left(\mathbf{H}_{\rho}, L_{2}\left(U, \mathbf{H}_{\rho}\right)\right), \forall v \in \mathbf{H}_{\rho}:\left(U \ni u \mapsto \mathbf{B}(v) u \in \mathbf{H}_{\gamma}\right) \in L\left(U, \mathbf{H}_{\gamma}\right)$, and $\left(\mathbf{H}_{\rho} \ni v \mapsto\left(U \ni u \mapsto \mathbf{B}(v) u \in \mathbf{H}_{\gamma}\right) \in L\left(U, \mathbf{H}_{\gamma}\right)\right) \in \operatorname{Lip}^{0}\left(\mathbf{H}_{\rho}, L\left(U, \mathbf{H}_{\gamma}\right)\right)$ and such that for all $\varepsilon \in(0, \infty), N \in \mathbb{N}$ it holds that

$$
c \cdot\left(\lambda_{N}\right)^{-\eta} \leq\left|\mathbb{E}\left[\varphi\left(\mathbf{X}_{T}^{\infty}\right)\right]-\mathbb{E}\left[\varphi\left(\mathbf{X}_{T}^{N}\right)\right]\right| \leq C_{\varepsilon} \cdot\left(\lambda_{N}\right)^{\varepsilon-\eta} .
$$

Further results on lower bounds for strong and weak errors for stochastic parabolic equations can be found, e.g., in Davie \& Gaines [15], Müller-Gronbach et al. [38], Müller-Gronbach \& Ritter [37], Conus et al. [9], and Jentzen \& Kurniawan [25].

Third, we illustrate Theorem 1.1 by a simple example (cf. Corollary 3.12). For this let $P_{N} \in L(H), N \in \mathbb{N} \cup\{\infty\}$, be the linear operators which satisfy for all $N \in \mathbb{N} \cup$ $\{\infty\}, v \in H$ that $P_{N}(v)=\sum_{n=1}^{N}\left\langle e_{n}, v\right\rangle_{H} e_{n}$. In the case where $\left(H,\langle\cdot, \cdot\rangle_{H},\|\cdot\|_{H}\right)=$ $\left(U,\langle\cdot, \cdot\rangle_{U},\|\cdot\|_{U}\right)=\left(L^{2}\left(\mu_{(0,1)} ; \mathbb{R}\right),\langle\cdot, \cdot\rangle_{L^{2}\left(\mu_{(0,1)} ; \mathbb{R}\right)},\|\cdot\|_{L^{2}\left(\mu_{(0,1)} ; \mathbb{R}\right)}\right), \xi=\left(\xi_{0}, \xi_{1}\right) \in$ $H_{0}^{1}((0,1) ; \mathbb{R}) \times H$, and $\mathbf{F}=0$, where $A: D(A) \subseteq H \rightarrow H$ is the Laplacian with Dirichlet boundary conditions on $H$, and where $\mathbf{B}: H \times H_{-1 / 2} \rightarrow L_{2}\left(H, H \times H_{-1 / 2}\right)$ is the function which satisfies for all $(v, w) \in H \times H_{-1 / 2}, u \in C([0,1], \mathbb{R})$ and $\mu_{(0,1)^{-}}$ a.e. $x \in(0,1)$ that $(\mathbf{B}(v, w) u)(x)=(0, v(x) u(x))$, the first component processes $X^{N}:[0, T] \times \Omega \rightarrow P_{N}(H), N \in \mathbb{N} \cup\{\infty\}$, are mild solutions of the stochastic partial differential equations

$$
\ddot{X}_{t}(x)=\frac{\partial^{2}}{\partial x^{2}} X_{t}(x)+P_{N} X_{t}(x) \dot{W}_{t}(x)
$$

with $X_{0}(x)=\left(P_{N} \xi_{0}\right)(x), \dot{X}_{0}(x)=\left(P_{N} \xi_{1}\right)(x)$, and $X_{t}(0)=X_{t}(1)=0$ for $x \in(0,1), t \in[0, T], N \in \mathbb{N} \cup\{\infty\}$. In the case $N=\infty,(1.4)$ is known as the 
continuous version of the hyperbolic Anderson model in the literature (cf., e.g., Conus et al. [10]). Theorem 1.1 applied to (1.4) ensures for all $\varphi \in C_{\mathrm{b}}^{2}(H, \mathbb{R}), \varepsilon \in(0, \infty)$ that there exists a real number $C \in[0, \infty)$ such that for all $N \in \mathbb{N}$ it holds that

$$
\left|\mathbb{E}\left[\varphi\left(X_{T}^{\infty}\right)\right]-\mathbb{E}\left[\varphi\left(X_{T}^{N}\right)\right]\right| \leq C \cdot N^{\varepsilon-1}
$$

(cf. Corollary 3.12). We thus prove that the spatial spectral Galerkin approximations converge with weak rate 1- to the solution of the continuous version of the hyperbolic Anderson model. The weak rate 1- is exactly twice the well-known strong convergence rate for the continuous version of the hyperbolic Anderson model. To the best of our knowledge, Theorem 1.1 is the first result in the literature that establishes an essentially sharp weak convergence rate for the continuous version of the hyperbolic Anderson model. Theorem 1.1 also establishes essentially sharp weak convergence rates for more general semilinear stochastic wave equations (cf. Corollaries 3.10 and 3.12 below).

The remainder of this article is organized as follows. In Sect. 1.1 some of the notation that is often used in this article is presented. Section 2.1 states mostly well-known existence, uniqueness, and regularity results, while Sect. 2.3 collects basic properties about interpolation spaces and semigroups associated to deterministic wave equations. The main result of this article, Theorem 3.6 below, is stated and proven in Sect. 3.2. It establishes upper bounds for weak errors for wave-type SEEs. Finally, Sect. 3.3 shows how this abstract result can be applied to stochastic wave equations and, in particular, to the continuous version of the hyperbolic Anderson model (cf. Corollaries 3.10 and 3.12 below).

\subsection{Notation}

In this section we introduce some notation which we employ throughout this article. For a set $A$ we denote by $\mathcal{P}(A)$ the power set of $A$ and by $\mathcal{P}_{0}(A)$ the set of all finite subsets of $A$. For a metric space $\left(E, d_{E}\right)$, a dense subset $A \subseteq E$, a complete metric space $\left(F, d_{F}\right)$, a uniformly continuous function $f: A \rightarrow F$, and the unique function $\tilde{f} \in C(E, F)$ which satisfies $\left.\tilde{f}\right|_{A}=f$ we often write, for simplicity of presentation, $f$ instead of $\tilde{f}$. For two $\mathbb{R}$-Banach spaces $\left(V,\|\cdot\|_{V}\right)$ and $\left(W,\|\cdot\|_{W}\right)$ with $V \neq\{0\}$, a natural number $k \in \mathbb{N}$, and a function $f \in C^{k}(V, W)$ we denote by $|f|_{C_{\mathrm{b}}^{k}(V, W)},\|f\|_{C_{\mathrm{b}}^{k}(V, W)} \in[0, \infty]$ the extended real numbers given by

$$
\begin{aligned}
|f|_{C_{\mathrm{b}}^{k}(V, W)} & =\sup _{x \in V}\left\|f^{(k)}(x)\right\|_{L^{(k)}(V, W)}=\sup _{x \in V} \sup _{v_{1}, \ldots, v_{k} \in V \backslash\{0\}} \frac{\left\|f^{(k)}(x)\left(v_{1}, \ldots, v_{k}\right)\right\|_{W}}{\left\|v_{1}\right\|_{V} \cdot \ldots \cdot\left\|v_{k}\right\|_{V}}, \\
\|f\|_{C_{\mathrm{b}}^{k}(V, W)} & =\|f(0)\|_{W}+\sum_{\ell=1}^{k}|f|_{C_{\mathrm{b}}^{\ell}(V, W)}
\end{aligned}
$$

and we denote by $C_{\mathrm{b}}^{k}(V, W)$ the set given by $C_{\mathrm{b}}^{k}(V, W)=\left\{g \in C^{k}(V, W):\|g\|_{C_{\mathrm{b}}^{k}(V, W)}\right.$ $<\infty\}$. For two $\mathbb{R}$-Banach spaces $\left(V,\|\cdot\|_{V}\right)$ and $\left(W,\|\cdot\|_{W}\right)$ with $V \neq\{0\}$, a number $k \in \mathbb{N}_{0}=\{0,1,2, \ldots\}$, and a function $f \in C^{k}(V, W)$ we denote by $|f|_{\operatorname{Lip}^{k}(V, W)},\|f\|_{\operatorname{Lip}^{k}(V, W)} \in[0, \infty]$ the extended real numbers given by 


$$
\begin{aligned}
|f|_{\operatorname{Lip}^{k}(V, W)} & = \begin{cases}\sup _{\substack{x, y \in V \\
x \neq y}}\left(\frac{\|f(x)-f(y)\|_{W}}{\|x-y\|_{V}}\right) & : k=0, \\
\sup _{\substack{x, y \in V \\
x \neq y}}\left(\frac{\left\|f^{(k)}(x)-f^{(k)}(y)\right\|_{L^{(k)}(V, W)}}{\|x-y\|_{V}}\right) & : k \in \mathbb{N},\end{cases} \\
\|f\|_{\operatorname{Lip}^{k}(V, W)} & =\|f(0)\|_{W}+\sum_{\ell=0}^{k}|f|_{\operatorname{Lip}^{\ell}(V, W)}
\end{aligned}
$$

and we denote by $\operatorname{Lip}^{k}(V, W)$ the set given by $\operatorname{Lip}^{k}(V, W)=\left\{g \in C^{k}(V, W)\right.$ : $\left.\|g\|_{\operatorname{Lip}^{k}(V, W)}<\infty\right\}$. For two $\mathbb{R}$-inner product spaces $\left(V,\langle\cdot, \cdot\rangle_{V},\|\cdot\|_{V}\right)$ and $\left(W,\langle\cdot, \cdot\rangle_{W},\|\cdot\|_{W}\right)$ we denote by $\left(V \times W,\langle\cdot, \cdot\rangle_{V \times W},\|\cdot\|_{V \times W}\right)$ the $\mathbb{R}$-inner product space which satisfies for all $x_{1}=\left(v_{1}, w_{1}\right), x_{2}=\left(v_{2}, w_{2}\right) \in V \times W$ that $\left\langle x_{1}, x_{2}\right\rangle_{V \times W}=\left\langle v_{1}, v_{2}\right\rangle_{V}+\left\langle w_{1}, w_{2}\right\rangle_{W}$. For $\mathbb{R}$-Hilbert spaces $\left(H_{i},\langle\cdot, \cdot\rangle_{H_{i}},\|\cdot\|_{H_{i}}\right)$, $i \in\{1,2\}$, a real number $p \in[1, \infty)$, and linear operators $A \in L\left(H_{1}, H_{2}\right)$ and $B \in L\left(H_{1}\right)$ we denote by $\|A\|_{L_{p}\left(H_{1}, H_{2}\right)} \in[0, \infty]$ the extended real number given by $\|A\|_{L_{p}\left(H_{1}, H_{2}\right)}=\left[\operatorname{trace}_{H_{1}}\left(\left(A^{\star} A\right)^{p / 2}\right)\right]^{1 / p}$, we denote by $\|B\|_{L_{p}\left(H_{1}\right)} \in[0, \infty]$ the extended real number given by $\|B\|_{L_{p}\left(H_{1}\right)}=\|B\|_{L_{p}\left(H_{1}, H_{1}\right)}$, we denote by $L_{p}\left(H_{1}, H_{2}\right)$ the set given by $L_{p}\left(H_{1}, H_{2}\right)=\left\{C \in L\left(H_{1}, H_{2}\right):\|C\|_{L_{p}\left(H_{1}, H_{2}\right)}<\infty\right\}$, and we denote by $L_{p}\left(H_{1}\right)$ the set given by $L_{p}\left(H_{1}\right)=L_{p}\left(H_{1}, H_{1}\right)$. For an $\mathbb{R}$ Hilbert space $\left(H,\langle\cdot, \cdot\rangle_{H},\|\cdot\|_{H}\right)$, an orthonormal basis $\mathbb{B} \subseteq H$ of $H$, a function $\lambda: \mathbb{B} \rightarrow \mathbb{R}$, a linear operator $A: D(A) \subseteq H \rightarrow H$ which satisfies $D(A)=$ $\left\{v \in H: \sum_{b \in \mathbb{B}}\left|\lambda_{b}\langle b, v\rangle_{H}\right|^{2}<\infty\right\}$ and $\forall v \in D(A): A v=\sum_{b \in \mathbb{B}} \lambda_{b}\langle b, v\rangle_{H} b$, and a function $\varphi: \mathbb{R} \rightarrow \mathbb{R}$ we denote by $\varphi(A): D(\varphi(A)) \subseteq H \rightarrow H$ the linear operator which satisfies $D(\varphi(A))=\left\{v \in H: \sum_{b \in \mathbb{B}}\left|\varphi\left(\lambda_{b}\right)\langle b, v\rangle_{H}\right|^{2}<\infty\right\}$ and $\forall v \in D(\varphi(A)): \varphi(A) v=\sum_{b \in \mathbb{B}} \varphi\left(\lambda_{b}\right)\langle b, v\rangle_{H} b$. For a Borel measurable set $A \in \mathcal{B}(\mathbb{R})$ we denote by $\mu_{A}: \mathcal{B}(A) \rightarrow[0, \infty]$ the Lebesgue-Borel measure on $A$.

\section{Preliminaries}

For the proof of our key results in Sect. 3 below we require a number of basic properties of solutions of Kolmogorov equations and of semigroups associated to wave-type evolution equations, which we collect in this section. More precisely, in Sect. 2.1 we state a well-known existence and uniqueness result for solutions of SEEs with Lipschitz continuous drift and diffusion coefficients (cf. Proposition 2.1 below) as well as an elementary result providing bounds for solutions of Kolmogorov equations associated to finite-dimensional SEEs (cf. Lemma 2.2 below). Furthermore, in Sect. 2.3 we recall several elementary and well-known facts about linear operators, semigroups, and interpolation spaces associated to deterministic linear wave-type evolution equations (cf. the setting in Sect. 2.2).

\subsection{Existence, Uniqueness, and Regularity Results for SEEs}

Proposition 2.1 below is a direct consequence of Theorem 7.4 in Da Prato \& Zabczyk [13]. 
Proposition 2.1 Consider the notation in Sect. 1.1, let $T \in(0, \infty), p \in[2, \infty)$, let $\left(H,\langle\cdot, \cdot\rangle_{H},\|\cdot\|_{H}\right)$ and $\left(U,\langle\cdot, \cdot\rangle_{U},\|\cdot\|_{U}\right)$ be separable $\mathbb{R}$-Hilbert spaces with $H \neq\{0\}$, let $(\Omega, \mathcal{F}, \mathbb{P})$ be a probability space with a normal filtration $\left(\mathbb{F}_{t}\right)_{t \in[0, T]}$, let $\left(W_{t}\right)_{t \in[0, T]}$ be an $\operatorname{id}_{U}$-cylindrical $\left(\Omega, \mathcal{F}, \mathbb{P},\left(\mathbb{F}_{t}\right)_{t \in[0, T]}\right)$-Wiener process, let $S:[0, \infty) \rightarrow L(H)$ be a strongly continuous semigroup, and let $F \in \operatorname{Lip}^{0}(H, H)$, $B \in \operatorname{Lip}^{0}\left(H, L_{2}(U, H)\right), \xi \in L^{p}\left(\left.\mathbb{P}\right|_{\mathbb{F}_{0}} ; H\right)$. Then there exists an up to modifications

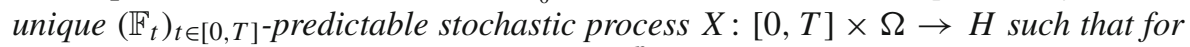
all $t \in[0, T]$ it holds that $\sup _{s \in[0, T]} \mathbb{E}\left[\left\|X_{S}\right\|_{H}^{p}\right]<\infty$ and $\mathbb{P}$-a.s. that

$$
X_{t}=S_{t} \xi+\int_{0}^{t} S_{t-s} F\left(X_{s}\right) \mathrm{d} s+\int_{0}^{t} S_{t-s} B\left(X_{s}\right) \mathrm{d} W_{s} .
$$

In the next elementary and well-known result, Lemma 2.2 below, we present bounds for spatial derivatives of solutions of Kolmogorov equations associated to finite-dimensional SEEs (cf., e.g., Lemma 2.3 in [23]).

Lemma 2.2 Consider the notation in Sect. 1.1, let $\left(H,\langle\cdot, \cdot\rangle_{H},\|\cdot\|_{H}\right)$ be a finitedimensional $\mathbb{R}$-vector space with $H \neq\{0\}$, let $\left(U,\langle\cdot, \cdot\rangle_{U},\|\cdot\|_{U}\right)$ be a separable $\mathbb{R}$-Hilbert space, let $\mathbb{U} \subseteq U$ be an orthonormal basis of $U$, let $T \in(0, \infty)$, $A \in L(H), F \in C_{\mathrm{b}}^{2}(H, H), B \in C_{\mathrm{b}}^{2}\left(H, L_{2}(U, H)\right), \varphi \in C_{\mathrm{b}}^{2}(H, \mathbb{R})$, let $(\Omega, \mathcal{F}, \mathbb{P})$ be a probability space with a normal filtration $\left(\mathbb{F}_{t}\right)_{t \in[0, T]}$, let $\left(W_{t}\right)_{t \in[0, T]}$ be an $\mathrm{id}_{U^{-}}$ cylindrical $\left(\Omega, \mathcal{F}, \mathbb{P},\left(\mathbb{F}_{t}\right)_{t \in[0, T]}\right)$-Wiener process, let $X^{x}:[0, T] \times \Omega \rightarrow H, x \in H$,

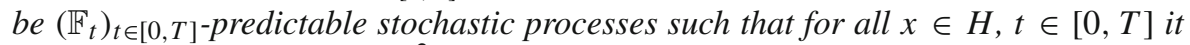
holds that $\sup _{s \in[0, T]} \mathbb{E}\left[\left\|X_{S}^{x}\right\|_{H}^{2}\right]<\infty$ and $\mathbb{P}$-a.s. that

$$
X_{t}^{x}=\mathrm{e}^{t A} x+\int_{0}^{t} \mathrm{e}^{(t-s) A} F\left(X_{s}^{x}\right) \mathrm{d} s+\int_{0}^{t} \mathrm{e}^{(t-s) A} B\left(X_{s}^{x}\right) \mathrm{d} W_{s},
$$

and let $u:[0, T] \times H \rightarrow \mathbb{R}$ be the function which satisfies for all $t \in[0, T], x \in H$ that $u(t, x)=\mathbb{E}\left[\varphi\left(X_{t}^{x}\right)\right]$. Then

(i) it holds that $u \in C^{1,2}([0, T] \times H, \mathbb{R})$,

(ii) it holds for all $(t, x) \in[0, T] \times H$ that

$$
\left(\frac{\partial}{\partial t} u\right)(t, x)=\left(\frac{\partial}{\partial x} u\right)(t, x)[A x+F(x)]+\frac{1}{2} \sum_{b \in \mathbb{U}}\left(\frac{\partial^{2}}{\partial x^{2}} u\right)(t, x)(B(x) b, B(x) b),
$$

(iii) it holds that

$$
\begin{aligned}
& \sup _{t \in[0, T]}|u(t, \cdot)|_{C_{\mathrm{b}}^{1}(H, \mathbb{R})} \leq|\varphi|_{C_{\mathrm{b}}^{1}(H, \mathbb{R})}\left[\sup _{s \in[0, T]}\left\|\mathrm{e}^{s A}\right\|_{L(H)}\right] \\
& \quad \cdot \exp \left(T\left[|F|_{C_{\mathrm{b}}^{1}(H, H)}+\frac{1}{2}|B|_{C_{\mathrm{b}}^{1}\left(H, L_{2}(U, H)\right)}^{2}\right] \sup _{s \in[0, T]}\left\|\mathrm{e}^{s A}\right\|_{L(H)}^{2}\right)<\infty,
\end{aligned}
$$

and 
(iv) it holds that

$$
\begin{aligned}
& \sup _{t \in[0, T]}|u(t, \cdot)|_{C_{\mathrm{b}}^{2}(H, \mathbb{R})} \\
& \leq\|\varphi\|_{C_{\mathrm{b}}^{2}(H, \mathbb{R})}\left[\sup _{s \in[0, T]}\left\|\mathrm{e}^{s A}\right\|_{L(H)}^{3}\right] \\
& \quad \max \left\{1,\left[T\left(|F|_{C_{\mathrm{b}}^{2}(H, H)}^{2}+2|B|_{C_{\mathrm{b}}^{2}\left(H, L_{2}(U, H)\right)}^{2}\right)\right]^{1 / 2}\right\} \\
& \quad \cdot \exp \left(T\left[\frac{1}{2}+3|F|_{C_{\mathrm{b}}^{1}(H, H)}+4|B|_{C_{\mathrm{b}}^{1}\left(H, L_{2}(U, H)\right)}^{2}\right] \sup _{s \in[0, T]}\left\|\mathrm{e}^{s A}\right\|_{L(H)}^{4}\right)<\infty .
\end{aligned}
$$

\subsection{Setting}

Consider the notation in Sect. 1.1 , let $\left(H,\langle\cdot, \cdot\rangle_{H},\|\cdot\|_{H}\right)$ be a separable $\mathbb{R}$-Hilbert space, let $\mathbb{H} \subseteq H$ be a nonempty orthonormal basis of $H$, let $\lambda: \mathbb{H} \rightarrow \mathbb{R}$ be a function which satisfies $\sup _{h \in \mathbb{H}} \lambda_{h}<0$, let $A: D(A) \subseteq H \rightarrow H$ be the linear operator which satisfies $D(A)=\left\{v \in H: \sum_{h \in \mathbb{H}}\left|\lambda_{h}\langle h, v\rangle_{H}\right|^{2}<\infty\right\}$ and $\forall v \in$ $D(A): A v=\sum_{h \in \mathbb{H}} \lambda_{h}\langle h, v\rangle_{H} h$, let $\left(H_{r},\langle\cdot, \cdot\rangle_{H_{r}},\|\cdot\|_{H_{r}}\right), r \in \mathbb{R}$, be a family of interpolation spaces associated to $-A$, let $\left(\mathbf{H}_{r},\langle\cdot, \cdot\rangle_{\mathbf{H}_{r}},\|\cdot\|_{\mathbf{H}_{r}}\right), r \in \mathbb{R}$, be the family of $\mathbb{R}$-Hilbert spaces which satisfies for all $r \in \mathbb{R}$ that $\left(\mathbf{H}_{r},\langle\cdot, \cdot\rangle_{\mathbf{H}_{r}},\|\cdot\|_{\mathbf{H}_{r}}\right)=\left(H_{r / 2} \times\right.$ $\left.H_{r / 2-1 / 2},\langle\cdot, \cdot\rangle_{H_{r / 2} \times H_{r / 2-1 / 2}},\|\cdot\|_{H_{r / 2} \times H_{r / 2-1 / 2}}\right)$, and let $\mathbf{A}: D(\mathbf{A}) \subseteq \mathbf{H}_{0} \rightarrow \mathbf{H}_{0}$ be the linear operator which satisfies $D(\mathbf{A})=\mathbf{H}_{1}$ and $\forall(v, w) \in \mathbf{H}_{1}: \mathbf{A}(v, w)=(w, A v)$.

\subsection{Basic Properties of Deterministic Linear Wave Equations}

The following elementary result, Lemma 2.3 below, provides a characterization for the family of $\mathbb{R}$-Hilbert spaces $\left(\mathbf{H}_{r},\langle\cdot, \cdot\rangle_{\mathbf{H}_{r}},\|\cdot\|_{\mathbf{H}_{r}}\right), r \in \mathbb{R}$, from the setting in Sect. 2.2 (cf., e.g., Lemma 2.4 in [23]).

Lemma 2.3 Assume the setting in Sect. 2.2 and let $\boldsymbol{\Lambda}: D(\boldsymbol{\Lambda}) \subseteq \mathbf{H}_{0} \rightarrow \mathbf{H}_{0}$ be the linear operator which satisfies for all $(v, w) \in \mathbf{H}_{1}$ that $D(\boldsymbol{\Lambda})=\mathbf{H}_{1}$ and

$$
\boldsymbol{\Lambda}(v, w)=\left(\begin{array}{c}
\sum_{h \in \mathbb{H}}\left|\lambda_{h}\right|^{1 / 2}\langle h, v\rangle_{H} h \\
\sum_{h \in \mathbb{H}}\left|\lambda_{h}\right|^{1 / 2}\langle h, w\rangle_{H} h
\end{array}\right) .
$$

Then the $\mathbb{R}$-Hilbert spaces $\left(\mathbf{H}_{r},\langle\cdot, \cdot\rangle_{\mathbf{H}_{r}},\|\cdot\|_{\mathbf{H}_{r}}\right), r \in \mathbb{R}$, are a family of interpolation spaces associated to $\boldsymbol{\Lambda}$.

The next elementary and well-known result, Lemma 2.4 below, can be found, e.g., in a slightly different form in Section 5.3 in Lindgren [33].

Lemma 2.4 Assume the setting in Sect. 2.2 and let $\mathbf{S}:[0, \infty) \rightarrow L\left(\mathbf{H}_{0}\right)$ be the function which satisfies for all $t \in[0, \infty),(v, w) \in \mathbf{H}_{0}$ that

$$
\mathbf{S}_{t}\left(\begin{array}{c}
v \\
w
\end{array}\right)=\left(\begin{array}{c}
\cos \left(t(-A)^{1 / 2}\right) v+(-A)^{-1 / 2} \sin \left(t(-A)^{1 / 2}\right) w \\
-(-A)^{1 / 2} \sin \left(t(-A)^{1 / 2}\right) v+\cos \left(t(-A)^{1 / 2}\right) w
\end{array}\right)
$$


Then $\mathbf{S}:[0, \infty) \rightarrow L\left(\mathbf{H}_{0}\right)$ is a strongly continuous semigroup of bounded linear operators on $\mathbf{H}_{0}$ and $\mathbf{A}: D(\mathbf{A}) \subseteq \mathbf{H}_{0} \rightarrow \mathbf{H}_{0}$ is the generator of $\mathbf{S}$.

The following two elementary and well-known assertions state that the semigroup in Lemma 2.4 above is a semigroup of isometries and that both this semigroup and its generator commute with Galerkin projections (cf., e.g., Lemmas 2.6 and 2.7 in [23]).

Lemma 2.5 Assume the setting in Sect. 2.2. Then

(i) it holds for all $t \in[0, \infty), x \in \mathbf{H}_{0}$ that $\left\|\mathrm{e}^{t \mathbf{A}} x\right\|_{\mathbf{H}_{0}}=\|x\|_{\mathbf{H}_{0}}$ and

(ii) it holds that $\sup _{t \in[0, \infty)}\left\|\mathrm{e}^{t \mathbf{A}}\right\|_{L\left(\mathbf{H}_{0}\right)}=1$.

Lemma 2.6 Assume the setting in Sect. 2.2 and let $\mathbf{P}_{I} \in L\left(\mathbf{H}_{0}\right), \quad I \in \mathcal{P}(\mathbb{H})$, be the linear operators which satisfy for all $I \in \mathcal{P}(\mathbb{H}), v, w \in H$ that $\mathbf{P}_{I}(v, w)=$ $\left(\sum_{h \in I}\langle h, v\rangle_{H} h, \sum_{h \in I}\langle h, w\rangle_{H} h\right)$. Then

(i) it holds for all $I \in \mathcal{P}(\mathbb{H}), x \in \mathbf{H}_{1}$ that $\mathbf{A P}_{I}(x)=\mathbf{P}_{I} \mathbf{A} x$ and

(ii) it holds for all $I \in \mathcal{P}(\mathbb{H}), t \in[0, \infty), x \in \mathbf{H}_{0}$ that $\mathrm{e}^{t \mathbf{A}} \mathbf{P}_{I}(x)=\mathbf{P}_{I} \mathrm{e}^{t \mathbf{A}} x$.

\section{Upper Bounds for Weak Errors}

In this section we establish upper bounds for weak errors associated to spatial spectral Galerkin approximations of semilinear stochastic wave equations; cf. Theorem 3.6 and Corollaries 3.7, 3.8, 3.10, and 3.12 below.

For many results in this section we consider an abstract setting of wave-type SEEs with appropriate Lipschitz and smoothness assumptions on the corresponding drift nonlinearity and diffusion coefficients; cf. the setting in Sect. 3.1 below. In Sect. 3.2 below we first present a suitable a priori estimate and a suitable perturbation estimate for solutions of certain wave-type SEEs; cf. Lemmas 3.2 and 3.3, respectively. Thereafter, we show an estimate for first and second order spatial derivatives of solutions to Kolmogorov equations associated to certain finite-dimensional wave-type SEEs; cf. Lemma 3.4 below. Following an elementary auxiliary lemma (cf. Lemma 3.5), we demonstrate the main theorem of this work, Theorem 3.6 below, which provides upper bounds for weak errors involving, among other terms, quantities depending on solutions of certain finite-dimensional wave-type SEEs as well as quantities depending on solutions of Kolmogorov equations associated to these SEEs (cf. also Corollary 3.7 below). Using the a priori estimate in Lemma 3.2 and the estimate for solutions of Kolmogorov equations in Lemma 3.4, the upper bounds in Theorem 3.6 are subsequently specialized in order to obtain upper bounds depending in an explicit way on the drift nonlinearity, the diffusion coefficient, and the initial value; cf. Corollary 3.8.

Finally, in Sect. 3.3 we apply Corollary 3.7 to prove essentially sharp weak convergence rates for spatial spectral Galerkin approximations of semilinear stochastic wave equations. In Corollary 3.10 we consider a setting with specialized drift nonlinearity but still quite general diffusion coefficient, while in Corollary 3.12 we consider a class of semilinear stochastic wave equations driven by multiplicative noise, that includes, in particular, the continuous version of the hyperbolic Anderson model. For 
the proofs of these results we recall two well-known facts about families of interpolation spaces associated to symmetric diagonal linear operators (cf., e.g., Sell \& You [40, Section 3.2]); cf. Lemmas 3.9 and 3.11 below.

\subsection{Setting}

Consider the notation in Sect. 1.1, let $\left(H,\langle\cdot, \cdot\rangle_{H},\|\cdot\|_{H}\right)$ and $\left(U,\langle\cdot, \cdot\rangle_{U},\|\cdot\|_{U}\right)$ be separable $\mathbb{R}$-Hilbert spaces, let $\mathbb{H} \subseteq H$ be a nonempty orthonormal basis of $H$, let $\lambda: \mathbb{H} \rightarrow \mathbb{R}$ be a function which satisfies $\sup _{h \in \mathbb{H}} \lambda_{h}<0$, let $A: D(A) \subseteq H \rightarrow H$ be the linear operator which satisfies $D(A)=\left\{v \in H: \sum_{h \in \mathbb{H}}\left|\lambda_{h}\langle h, v\rangle_{H}\right|^{2}<\infty\right\}$ and $\forall v \in D(A): A v=\sum_{h \in \mathbb{H}} \lambda_{h}\langle h, v\rangle_{H} h$, let $\left(H_{r},\langle\cdot, \cdot\rangle_{H_{r}},\|\cdot\|_{H_{r}}\right), r \in \mathbb{R}$, be a family of interpolation spaces associated to $-A$, let $\left(\mathbf{H}_{r},\langle\cdot, \cdot\rangle_{\mathbf{H}_{r}},\|\cdot\|_{\mathbf{H}_{r}}\right), r \in \mathbb{R}$, be the family of $\mathbb{R}$-Hilbert spaces which satisfies for all $r \in \mathbb{R}$ that $\left(\mathbf{H}_{r},\langle\cdot, \cdot\rangle_{\mathbf{H}_{r}},\|\cdot\|_{\mathbf{H}_{r}}\right)=$ $\left(H_{r / 2} \times H_{r / 2-1 / 2},\langle\cdot, \cdot\rangle_{H_{r / 2} \times H_{r / 2-1 / 2}},\|\cdot\|_{H_{r / 2} \times H_{r / 2-1 / 2}}\right)$, let $\mathbf{P}_{I} \in L\left(\mathbf{H}_{0}\right), I \in \mathcal{P}(\mathbb{H})$, be the linear operators which satisfy for all $I \in \mathcal{P}(\mathbb{H}), v, w \in H$ that $\mathbf{P}_{I}(v, w)=$ $\left(\sum_{h \in I}\langle h, v\rangle_{H} h, \sum_{h \in I}\langle h, w\rangle_{H} h\right)$, let $\mathbf{A}: D(\mathbf{A}) \subseteq \mathbf{H}_{0} \rightarrow \mathbf{H}_{0}$ be the linear operator which satisfies $D(\mathbf{A})=\mathbf{H}_{1}$ and $\forall(v, w) \in \mathbf{H}_{1}: \mathbf{A}(v, w)=(w, A v)$, let $\boldsymbol{\Lambda}: D(\boldsymbol{\Lambda}) \subseteq \mathbf{H}_{0} \rightarrow \mathbf{H}_{0}$ be the linear operator which satisfies $D(\boldsymbol{\Lambda})=\mathbf{H}_{1}$ and $\forall(v, w) \in \mathbf{H}_{1}: \boldsymbol{\Lambda}(v, w)=\left(\sum_{h \in \mathbb{H}}\left|\lambda_{h}\right|^{1 / 2}\langle h, v\rangle_{H} h, \sum_{h \in \mathbb{H}}\left|\lambda_{h}\right|^{1 / 2}\langle h, w\rangle_{H} h\right)$, let $T \in(0, \infty)$, let $(\Omega, \mathcal{F}, \mathbb{P})$ be a probability space with a normal filtration $\left(\mathbb{F}_{t}\right)_{t \in[0, T]}$, let $\left(W_{t}\right)_{t \in[0, T]}$ be an $\operatorname{id}_{U}$-cylindrical $\left(\Omega, \mathcal{F}, \mathbb{P},\left(\mathbb{F}_{t}\right)_{t \in[0, T]}\right)$-Wiener process, and let $\gamma \in(0, \infty), \beta \in(\gamma / 2, \gamma], \rho \in[0,2(\gamma-\beta)], \varrho, C_{\mathbf{F}}, C_{\mathbf{B}} \in[0, \infty)$, $\xi \in L^{2}\left(\left.\mathbb{P}\right|_{\mathbb{F}_{0}} ; \mathbf{H}_{2(\gamma-\beta)}\right), \mathbf{F} \in \operatorname{Lip}^{0}\left(\mathbf{H}_{0}, \mathbf{H}_{0}\right), \mathbf{B} \in \operatorname{Lip}^{0}\left(\mathbf{H}_{0}, L_{2}\left(U, \mathbf{H}_{0}\right)\right)$ satisfy $\boldsymbol{\Lambda}^{-\beta} \in$ $L_{2}\left(\mathbf{H}_{0}\right), \mathbf{F}\left(\mathbf{H}_{\rho}\right) \subseteq \mathbf{H}_{2(\gamma-\beta)},\left(\mathbf{H}_{\rho} \ni v \mapsto \mathbf{F}(v) \in \mathbf{H}_{2(\gamma-\beta)}\right) \in \operatorname{Lip}^{0}\left(\mathbf{H}_{\rho}, \mathbf{H}_{2(\gamma-\beta)}\right)$, $\forall v \in \mathbf{H}_{\rho}, u \in U: \mathbf{B}(v) u \in \mathbf{H}_{\gamma}, \forall v \in \mathbf{H}_{\rho}:\left(U \ni u \mapsto \mathbf{B}(v) u \in \mathbf{H}_{\rho}\right) \in L_{2}\left(U, \mathbf{H}_{\rho}\right)$, $\left(\mathbf{H}_{\rho} \ni v \mapsto\left(U \ni u \mapsto \mathbf{B}(v) u \in \mathbf{H}_{\rho}\right) \in L_{2}\left(U, \mathbf{H}_{\rho}\right)\right) \in \operatorname{Lip}^{0}\left(\mathbf{H}_{\rho}, L_{2}\left(U, \mathbf{H}_{\rho}\right)\right)$, $\forall v \in \mathbf{H}_{\rho}:\left(U \ni u \mapsto \mathbf{B}(v) u \in \mathbf{H}_{\gamma}\right) \in L\left(U, \mathbf{H}_{\gamma}\right),\left(\mathbf{H}_{\rho} \ni v \mapsto(U \ni u \mapsto \mathbf{B}(v) u \in\right.$ $\left.\left.\mathbf{H}_{\gamma}\right) \in L\left(U, \mathbf{H}_{\gamma}\right)\right) \in \operatorname{Lip}^{0}\left(\mathbf{H}_{\rho}, L\left(U, \mathbf{H}_{\gamma}\right)\right),\left.\mathbf{F}\right|_{\mathbf{H}_{\varrho}} \in C^{2}\left(\mathbf{H}_{\varrho}, \mathbf{H}_{0}\right),\left.\mathbf{B}\right|_{\mathbf{H}_{\varrho}} \in C^{2}\left(\mathbf{H}_{\varrho}\right.$, $\left.L_{2}\left(U, \mathbf{H}_{0}\right)\right), C_{\mathbf{F}}=\sup _{x, v_{1}, v_{2} \in \cap_{r \in \mathbb{R}} \mathbf{H}_{r}, \max \left\{\left\|v_{1}\right\|_{\mathbf{H}_{0}},\left\|v_{2}\right\|_{\mathbf{H}_{0}}\right\} \leq 1}\left\|\mathbf{F}^{\prime \prime}(x)\left(v_{1}, v_{2}\right)\right\|_{\mathbf{H}_{0}}<\infty$, and $C_{\mathbf{B}}=\sup _{x, v_{1}, v_{2} \in \cap_{r \in \mathbb{R}} \mathbf{H}_{r}, \max \left\{\left\|v_{1}\right\|_{\mathbf{H}_{0}},\left\|v_{2}\right\|_{\mathbf{H}_{0}}\right\} \leq 1}\left\|\mathbf{B}^{\prime \prime}(x)\left(v_{1}, v_{2}\right)\right\|_{L_{2}\left(U, \mathbf{H}_{0}\right)}<\infty$.

\subsection{Weak Convergence Rates for Galerkin Approximations}

Remark 3.1 Assume the setting in Sect. 3.1. Note that the assumption that $\left(\mathbf{H}_{\rho} \ni\right.$ $\left.v \mapsto \mathbf{F}(v) \in \mathbf{H}_{2(\gamma-\beta)}\right) \in \operatorname{Lip}^{0}\left(\mathbf{H}_{\rho}, \mathbf{H}_{2(\gamma-\beta)}\right)$ ensures that $\left(\mathbf{H}_{\rho} \ni v \mapsto \mathbf{F}(v) \in\right.$ $\left.\mathbf{H}_{\rho}\right) \in \operatorname{Lip}^{0}\left(\mathbf{H}_{\rho}, \mathbf{H}_{\rho}\right)$. The assumption that $\left(\mathbf{H}_{\rho} \ni v \mapsto\left(U \ni u \mapsto \mathbf{B}(v) u \in \mathbf{H}_{\rho}\right) \in\right.$ $\left.L_{2}\left(U, \mathbf{H}_{\rho}\right)\right) \in \operatorname{Lip}^{0}\left(\mathbf{H}_{\rho}, L_{2}\left(U, \mathbf{H}_{\rho}\right)\right)$ and Proposition 2.1 hence show that there exist up to modifications unique $\left(\mathbb{F}_{t}\right)_{t \in[0, T]}$-predictable stochastic processes $X^{I}:[0, T] \times$ $\Omega \rightarrow \mathbf{P}_{I}\left(\mathbf{H}_{\rho}\right), I \in \mathcal{P}(\mathbb{H})$, such that for all $I \in \mathcal{P}(\mathbb{H}), t \in[0, T]$ it holds that $\sup _{s \in[0, T]} \mathbb{E}\left[\left\|X_{s}^{I}\right\|_{\mathbf{H}_{\rho}}^{2}\right]<\infty$ and $\mathbb{P}$-a.s. that

$$
X_{t}^{I}=\mathrm{e}^{t \mathbf{A}} \mathbf{P}_{I} \xi+\int_{0}^{t} \mathrm{e}^{(t-s) \mathbf{A}} \mathbf{P}_{I} \mathbf{F}\left(X_{s}^{I}\right) \mathrm{d} s+\int_{0}^{t} \mathrm{e}^{(t-s) \mathbf{A}} \mathbf{P}_{I} \mathbf{B}\left(X_{s}^{I}\right) \mathrm{d} W_{s} .
$$


The next elementary result, Lemma 3.2, provides global a priori $L^{2}$-bounds for the stochastic processes $X^{I}:[0, T] \times \Omega \rightarrow \mathbf{P}_{I}\left(\mathbf{H}_{\rho}\right), I \in \mathcal{P}(\mathbb{H})$, from Remark 3.1 (cf., e.g., Lemma 3.2 in [23]).

Lemma 3.2 Assume the setting in Sect. 3.1 and let $X^{I}:[0, T] \times \Omega \rightarrow \mathbf{P}_{I}\left(\mathbf{H}_{\rho}\right), I \in$

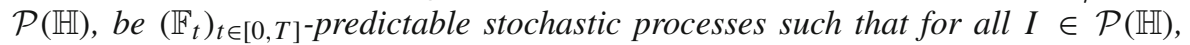
$t \in[0, T]$ it holds that $\sup _{s \in[0, T]} \mathbb{E}\left[\left\|X_{s}^{I}\right\|_{\mathbf{H}_{\rho}}^{2}\right]<\infty$ and $\mathbb{P}$-a.s. that

$$
X_{t}^{I}=\mathrm{e}^{t \mathbf{A}} \mathbf{P}_{I} \xi+\int_{0}^{t} \mathrm{e}^{(t-s) \mathbf{A}} \mathbf{P}_{I} \mathbf{F}\left(X_{s}^{I}\right) \mathrm{d} s+\int_{0}^{t} \mathrm{e}^{(t-s) \mathbf{A}} \mathbf{P}_{I} \mathbf{B}\left(X_{s}^{I}\right) \mathrm{d} W_{s} .
$$

Then

$$
\begin{aligned}
& \sup _{I \in \mathcal{P}(\mathbb{H})} \sup _{t \in[0, T]} \max \left\{1, \mathbb{E}\left[\left\|X_{t}^{I}\right\|_{\mathbf{H}_{\rho}}^{2}\right]\right\} \leq \max \left\{1, \mathbb{E}\left[\|\xi\|_{\mathbf{H}_{\rho}}^{2}\right]\right\} \\
& \cdot \exp \left(T\left[2\left\|\left.\mathbf{F}\right|_{\mathbf{H}_{\rho}}\right\|_{\operatorname{Lip}^{0}\left(\mathbf{H}_{\rho}, \mathbf{H}_{\rho}\right)}+\left\|\left.\mathbf{B}\right|_{\mathbf{H}_{\rho}}\right\|_{\operatorname{Lip}^{0}\left(\mathbf{H}_{\rho}, L_{2}\left(U, \mathbf{H}_{\rho}\right)\right)}^{2}\right]\right)<\infty .
\end{aligned}
$$

In the next result, Lemma 3.3 below, we present an elementary perturbation estimate (cf., e.g., Lemma 3.3 in [23]).

Lemma 3.3 Assume the setting in Sect. 3.1 and let $X^{I}:[0, T] \times \Omega \rightarrow \mathbf{P}_{I}\left(\mathbf{H}_{0}\right), I \in$

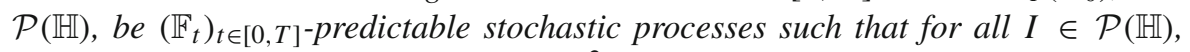
$t \in[0, T]$ it holds that $\sup _{s \in[0, T]} \mathbb{E}\left[\left\|X_{s}^{I}\right\|_{\mathbf{H}_{0}}^{2}\right]<\infty$ and $\mathbb{P}$-a.s. that

$$
X_{t}^{I}=\mathrm{e}^{t \mathbf{A}} \mathbf{P}_{I} \xi+\int_{0}^{t} \mathrm{e}^{(t-s) \mathbf{A}} \mathbf{P}_{I} \mathbf{F}\left(X_{s}^{I}\right) \mathrm{d} s+\int_{0}^{t} \mathrm{e}^{(t-s) \mathbf{A}} \mathbf{P}_{I} \mathbf{B}\left(X_{s}^{I}\right) \mathrm{d} W_{s} .
$$

Then it holds for all $I, J \in \mathcal{P}(\mathbb{H})$ that

$$
\begin{aligned}
& \sup _{t \in[0, T]} \mathbb{E}\left[\left\|X_{t}^{I}-X_{t}^{J}\right\|_{\mathbf{H}_{0}}^{2}\right] \leq 2\left[\sup _{t \in[0, T]} \mathbb{E}\left[\left\|\mathbf{P}_{I \backslash J} X_{t}^{I}+\mathbf{P}_{J \backslash I} X_{t}^{J}\right\|_{\mathbf{H}_{0}}^{2}\right]\right] \\
& \quad \cdot \exp \left(\left[\sqrt{2} T\left|\mathbf{P}_{I \cap J} \mathbf{F}\right|_{\operatorname{Lip}^{0}\left(\mathbf{H}_{0}, \mathbf{H}_{0}\right)}+\sqrt{2 T}\left|\mathbf{P}_{I \cap J} \mathbf{B}\right|_{\operatorname{Lip}^{0}\left(\mathbf{H}_{0}, L_{2}\left(U, \mathbf{H}_{0}\right)\right)}\right]^{2}\right)<\infty .
\end{aligned}
$$

Lemma 3.4 Assume the setting in Sect. 3.1, let $X^{J, x}:[0, T] \times \Omega \rightarrow \mathbf{P}_{J}\left(\mathbf{H}_{0}\right), x \in$ $\mathbf{P}_{J}\left(\mathbf{H}_{0}\right), J \in \mathcal{P}_{0}(\mathbb{H})$, be $\left(\mathbb{F}_{t}\right)_{t \in[0, T] \text {-predictable stochastic processes such that for all }}$ $J \in \mathcal{P}_{0}(\mathbb{H}), x \in \mathbf{P}_{J}\left(\mathbf{H}_{0}\right), t \in[0, T]$ it holds that $\sup _{s \in[0, T]} \mathbb{E}\left[\left\|X_{s}^{J, x}\right\|_{\mathbf{H}_{0}}^{2}\right]<\infty$ and $\mathbb{P}$-a.s. that

$$
X_{t}^{J, x}=\mathrm{e}^{t \mathbf{A}} x+\int_{0}^{t} \mathrm{e}^{(t-s) \mathbf{A}} \mathbf{P}_{J} \mathbf{F}\left(X_{s}^{J, x}\right) \mathrm{d} s+\int_{0}^{t} \mathrm{e}^{(t-s) \mathbf{A}} \mathbf{P}_{J} \mathbf{B}\left(X_{s}^{J, x}\right) \mathrm{d} W_{s},
$$

let $\varphi \in C_{\mathrm{b}}^{2}\left(\mathbf{H}_{0}, \mathbb{R}\right)$, and let $u^{J}:[0, T] \times \mathbf{P}_{J}\left(\mathbf{H}_{0}\right) \rightarrow \mathbb{R}, J \in \mathcal{P}_{0}(\mathbb{H})$, be the functions which satisfyfor all $J \in \mathcal{P}_{0}(\mathbb{H}),(t, x) \in[0, T] \times \mathbf{P}_{J}\left(\mathbf{H}_{0}\right)$ that $^{J}(t, x)=\mathbb{E}\left[\varphi\left(X_{t}^{J, x}\right)\right]$. Then 
(i) it holds for all $J \in \mathcal{P}_{0}(\mathbb{H})$ that $u^{J} \in C^{1,2}\left([0, T] \times \mathbf{P}_{J}\left(\mathbf{H}_{0}\right), \mathbb{R}\right)$,

(ii) it holds that

$$
\begin{aligned}
& \sup _{J \in \mathcal{P}_{0}(\mathbb{H})} \sup _{t \in[0, T]}\left|u^{J}(t, \cdot)\right|_{C_{\mathrm{b}}^{1}\left(\mathbf{P}_{J}\left(\mathbf{H}_{0}\right), \mathbb{R}\right)} \\
& \leq|\varphi|_{C_{\mathrm{b}}^{1}\left(\mathbf{H}_{0}, \mathbb{R}\right)} \exp \left(T\left[|\mathbf{F}|_{\operatorname{Lip}^{0}\left(\mathbf{H}_{0}, \mathbf{H}_{0}\right)}+\frac{1}{2}|\mathbf{B}|_{\operatorname{Lip}^{0}\left(\mathbf{H}_{0}, L_{2}\left(U, \mathbf{H}_{0}\right)\right)}^{2}\right]\right)<\infty,
\end{aligned}
$$

and

(iii) it holds that

$$
\begin{aligned}
& \sup _{J \in \mathcal{P}_{0}(\mathbb{H})} \sup _{t \in[0, T]}\left|u^{J}(t, \cdot)\right|_{C_{\mathrm{b}}^{2}\left(\mathbf{P}_{J}\left(\mathbf{H}_{0}\right), \mathbb{R}\right)} \leq\|\varphi\|_{C_{\mathrm{b}}^{2}\left(\mathbf{H}_{0}, \mathbb{R}\right)} \max \left\{1,\left[T\left(\left(C_{\mathbf{F}}\right)^{2}+2\left(C_{\mathbf{B}}\right)^{2}\right)\right]^{1 / 2}\right\} \\
& \cdot \exp \left(T\left[\frac{1}{2}+3|\mathbf{F}|_{\operatorname{Lip}^{0}\left(\mathbf{H}_{0}, \mathbf{H}_{0}\right)}+4|\mathbf{B}|_{\operatorname{Lip}^{0}\left(\mathbf{H}_{0}, L_{2}\left(U, \mathbf{H}_{0}\right)\right)}^{2}\right]\right)<\infty .
\end{aligned}
$$

Proof of Lemma 3.4 Observe that it holds for all $J \in \mathcal{P}_{0}(\mathbb{H})$ that $\mathbf{P}_{J}\left(\mathbf{H}_{0}\right) \subseteq\left(\bigcap_{r \in \mathbb{R}} \mathbf{H}_{r}\right)$ is a finite-dimensional $\mathbb{R}$-vector space. The assumptions that $\left.\mathbf{F}\right|_{\mathbf{H}_{\varrho}} \in C^{2}\left(\mathbf{H}_{\varrho}, \mathbf{H}_{0}\right)$, $\left.\mathbf{B}\right|_{\mathbf{H}_{\varrho}} \in C^{2}\left(\mathbf{H}_{\varrho}, L_{2}\left(U, \mathbf{H}_{0}\right)\right), \mathbf{F} \in \operatorname{Lip}^{0}\left(\mathbf{H}_{0}, \mathbf{H}_{0}\right), \mathbf{B} \in \operatorname{Lip}^{0}\left(\mathbf{H}_{0}, L_{2}\left(U, \mathbf{H}_{0}\right)\right)$, and $C_{\mathbf{F}}+C_{\mathbf{B}}<\infty$ hence ensure for all $J \in \mathcal{P}_{0}(\mathbb{H})$ that $\left(\mathbf{P}_{J}\left(\mathbf{H}_{0}\right) \ni v \mapsto \mathbf{P}_{J} \mathbf{F}(v) \in\right.$ $\left.\mathbf{P}_{J}\left(\mathbf{H}_{0}\right)\right) \in C_{\mathrm{b}}^{2}\left(\mathbf{P}_{J}\left(\mathbf{H}_{0}\right), \mathbf{P}_{J}\left(\mathbf{H}_{0}\right)\right)$ and $\left(\mathbf{P}_{J}\left(\mathbf{H}_{0}\right) \ni v \mapsto\left(U \ni u \mapsto \mathbf{P}_{J} \mathbf{B}(v) u \in\right.\right.$ $\left.\left.\mathbf{P}_{J}\left(\mathbf{H}_{0}\right)\right) \in L_{2}\left(U, \mathbf{P}_{J}\left(\mathbf{H}_{0}\right)\right)\right) \in C_{\mathrm{b}}^{2}\left(\mathbf{P}_{J}\left(\mathbf{H}_{0}\right), L_{2}\left(U, \mathbf{P}_{J}\left(\mathbf{H}_{0}\right)\right)\right)$. Therefore, Lemmas 2.2 and 2.5 prove for all $J \in \mathcal{P}_{0}(\mathbb{H})$ that $u^{J} \in C^{1,2}\left([0, T] \times \mathbf{P}_{J}\left(\mathbf{H}_{0}\right), \mathbb{R}\right)$,

$$
\begin{aligned}
& \sup _{t \in[0, T]}\left|u^{J}(t, \cdot)\right|_{C_{\mathrm{b}}^{1}\left(\mathbf{P}_{J}\left(\mathbf{H}_{0}\right), \mathbb{R}\right)} \leq\left.|\varphi|_{\mathbf{P}_{J}\left(\mathbf{H}_{0}\right)}\right|_{C_{\mathrm{b}}^{1}\left(\mathbf{P}_{J}\left(\mathbf{H}_{0}\right), \mathbb{R}\right)} \\
& \quad \cdot \exp \left(T\left[\left.\left|\mathbf{P}_{J} \mathbf{F}\right|_{\mathbf{P}_{J}\left(\mathbf{H}_{0}\right)}\right|_{C_{\mathrm{b}}^{1}\left(\mathbf{P}_{J}\left(\mathbf{H}_{0}\right), \mathbf{P}_{J}\left(\mathbf{H}_{0}\right)\right)}+\left.\frac{1}{2}\left|\mathbf{P}_{J} \mathbf{B}\right| \mathbf{P}_{J}\left(\mathbf{H}_{0}\right)\right|_{C_{\mathrm{b}}^{1}\left(\mathbf{P}_{J}\left(\mathbf{H}_{0}\right), L_{2}\left(U, \mathbf{P}_{J}\left(\mathbf{H}_{0}\right)\right)\right)} ^{2}\right]\right),
\end{aligned}
$$

and

$$
\begin{aligned}
& \sup _{t \in[0, T]}\left|u^{J}(t, \cdot)\right|_{C_{\mathrm{b}}^{2}\left(\mathbf{P}_{J}\left(\mathbf{H}_{0}\right), \mathbb{R}\right)} \leq\left\|\left.\varphi\right|_{\mathbf{P}_{J}\left(\mathbf{H}_{0}\right)}\right\|_{C_{\mathrm{b}}^{2}\left(\mathbf{P}_{J}\left(\mathbf{H}_{0}\right), \mathbb{R}\right)} \\
& \cdot \max \left\{1,\left[T \left(\left.\left|\mathbf{P}_{J} \mathbf{F}\right| \mathbf{P}_{J}\left(\mathbf{H}_{0}\right)\right|_{C_{\mathrm{b}}^{2}\left(\mathbf{P}_{J}\left(\mathbf{H}_{0}\right), \mathbf{P}_{J}\left(\mathbf{H}_{0}\right)\right)} ^{2}\right.\right.\right. \\
& \left.\left.\left.+\left.2\left|\mathbf{P}_{J} \mathbf{B}\right| \mathbf{P}_{J}\left(\mathbf{H}_{0}\right)\right|_{C_{\mathrm{b}}^{2}\left(\mathbf{P}_{J}\left(\mathbf{H}_{0}\right), L_{2}\left(U, \mathbf{P}_{J}\left(\mathbf{H}_{0}\right)\right)\right)} ^{2}\right)\right]^{1 / 2}\right\} \\
& \cdot \exp \left(T \left[\frac{1}{2}+\left.3\left|\mathbf{P}_{J} \mathbf{F}\right| \mathbf{P}_{J}\left(\mathbf{H}_{0}\right)\right|_{C_{\mathrm{b}}^{1}\left(\mathbf{P}_{J}\left(\mathbf{H}_{0}\right), \mathbf{P}_{J}\left(\mathbf{H}_{0}\right)\right)}\right.\right. \\
& \left.\left.+\left.4\left|\mathbf{P}_{J} \mathbf{B}\right|_{\mathbf{P}_{J}\left(\mathbf{H}_{0}\right)}\right|_{C_{\mathrm{b}}^{1}\left(\mathbf{P}_{J}\left(\mathbf{H}_{0}\right), L_{2}\left(U, \mathbf{P}_{J}\left(\mathbf{H}_{0}\right)\right)\right)} ^{2}\right]\right)<\infty \text {. }
\end{aligned}
$$

This implies (i)-(iii) and thus completes the proof of Lemma 3.4.

Before we present the main result of this article, Theorem 3.6 below, we recall the following elementary and well-known lemma, which is employed in the proof of Theorem 3.6.

Lemma 3.5 Let $p \in[0, \infty)$, let $\mathcal{J}_{n}, n \in \mathbb{N}_{0}$, be sets which satisfy for all $n \in \mathbb{N}$ that $\mathcal{J}_{n} \subseteq \mathcal{J}_{n+1}$ and $\bigcup_{m=1}^{\infty} \mathcal{J}_{m}=\mathcal{J}_{0}$, and let $g: \mathcal{J}_{0} \rightarrow(0, \infty)$ be a function which satisfies $\sum_{h \in \mathcal{J}_{0}}\left(g_{h}\right)^{p}<\infty$. Then 


$$
\underset{n \rightarrow \infty}{\limsup \sup }\left(\left\{g_{h}: h \in \mathcal{J}_{0} \backslash \mathcal{J}_{n}\right\} \cup\{0\}\right)=0 .
$$

Proof of Lemma 3.5 Without loss of generality we assume that $p \in(0, \infty)$ (otherwise (3.11) is clear). Observe that for all $n \in \mathbb{N}$ it holds that

$$
\left[\sup \left(\left\{g_{h}: h \in \mathcal{J}_{0} \backslash \mathcal{J}_{n}\right\} \cup\{0\}\right)\right]^{p} \leq \sum_{h \in \mathcal{J}_{0} \backslash \mathcal{J}_{n}}\left(g_{h}\right)^{p}=\sum_{h \in \mathcal{J}_{0}}\left(g_{h}\right)^{p}-\sum_{h \in \mathcal{J}_{n}}\left(g_{h}\right)^{p} .
$$

Moreover, note that Lebesgue's theorem of dominated convergence proves that

$$
\limsup _{n \rightarrow \infty}\left[\sum_{h \in \mathcal{J}_{0}}\left(g_{h}\right)^{p}-\sum_{h \in \mathcal{J}_{n}}\left(g_{h}\right)^{p}\right]=0
$$

Combining this with (3.12) completes the proof of Lemma 3.5.

Theorem 3.6 Assume the setting in Sect. 3.1, let $X^{I}:[0, T] \times \Omega \rightarrow \mathbf{P}_{I}\left(\mathbf{H}_{\rho}\right), I \in$ $\mathcal{P}(\mathbb{H})$, and $X^{J, x}:[0, T] \times \Omega \rightarrow \mathbf{P}_{J}\left(\mathbf{H}_{0}\right), x \in \mathbf{P}_{J}\left(\mathbf{H}_{0}\right), J \in \mathcal{P}_{0}(\mathbb{H})$, be $\left(\mathbb{F}_{t}\right)_{t \in[0, T]^{-}}$ predictable stochastic processes such that for all $I \in \mathcal{P}(\mathbb{H}), J \in \mathcal{P}_{0}(\mathbb{H}), x \in \mathbf{P}_{J}\left(\mathbf{H}_{0}\right)$, $t \in[0, T]$ it holds that $\sup _{s \in[0, T]} \mathbb{E}\left[\left\|X_{s}^{I}\right\|_{\mathbf{H}_{\rho}}^{2}+\left\|X_{s}^{J, x}\right\|_{\mathbf{H}_{0}}^{2}\right]<\infty$ and $\mathbb{P}$-a.s. that

$$
\begin{aligned}
X_{t}^{I} & =\mathrm{e}^{t \mathbf{A}} \mathbf{P}_{I} \xi+\int_{0}^{t} \mathrm{e}^{(t-s) \mathbf{A}} \mathbf{P}_{I} \mathbf{F}\left(X_{s}^{I}\right) \mathrm{d} s+\int_{0}^{t} \mathrm{e}^{(t-s) \mathbf{A}} \mathbf{P}_{I} \mathbf{B}\left(X_{s}^{I}\right) \mathrm{d} W_{s}, \\
X_{t}^{J, x} & =\mathrm{e}^{t \mathbf{A}} x+\int_{0}^{t} \mathrm{e}^{(t-s) \mathbf{A}} \mathbf{P}_{J} \mathbf{F}\left(X_{s}^{J, x}\right) \mathrm{d} s+\int_{0}^{t} \mathrm{e}^{(t-s) \mathbf{A}} \mathbf{P}_{J} \mathbf{B}\left(X_{s}^{J, x}\right) \mathrm{d} W_{s},
\end{aligned}
$$

let $\varphi \in C_{\mathrm{b}}^{2}\left(\mathbf{H}_{0}, \mathbb{R}\right)$, and let $u^{J}:[0, T] \times \mathbf{P}_{J}\left(\mathbf{H}_{0}\right) \rightarrow \mathbb{R}, J \in \mathcal{P}_{0}(\mathbb{H})$, be the functions which satisfy for all $J \in \mathcal{P}_{0}(\mathbb{H}),(t, x) \in[0, T] \times \mathbf{P}_{J}\left(\mathbf{H}_{0}\right)$ that $u^{J}(t, x)=\mathbb{E}\left[\varphi\left(X_{t}^{J, x}\right)\right]$. Then it holds for all $I \in \mathcal{P}(\mathbb{H}) \backslash\{\mathbb{H}\}$ that

$$
\begin{aligned}
& \left|\mathbb{E}\left[\varphi\left(X_{T}^{\mathbb{H}}\right)\right]-\mathbb{E}\left[\varphi\left(X_{T}^{I}\right)\right]\right| \\
& \leq\left(\left[\sup _{\left.J \in \mathcal{P}_{0}(\mathbb{H})\right)} \sup _{t \in[0, T]}\left|u^{J}(t, \cdot)\right|_{C_{\mathrm{b}}^{1}\left(\mathbf{P}_{J}\left(\mathbf{H}_{0}\right), \mathbb{R}\right)}\right]\right. \\
& \cdot\left[\mathbb{E}\left[\|\xi\|_{\mathbf{H}_{2(\gamma-\beta)}}\right]+\sup _{J \in \mathcal{P}_{0}(\mathbb{H})} \int_{0}^{T} \mathbb{E}\left[\left\|\mathbf{F}\left(X_{s}^{J}\right)\right\|_{\mathbf{H}_{2(\gamma-\beta)}}\right] \mathrm{d} s\right] \\
& +\left\|\boldsymbol{\Lambda}^{-\beta}\right\|_{L_{2}\left(\mathbf{H}_{0}\right)}^{2}\left[\sup _{J \in \mathcal{P}_{0}(\mathbb{H})} \sup _{t \in[0, T]}\left|u^{J}(t, \cdot)\right|_{C_{\mathrm{b}}^{2}\left(\mathbf{P}_{J}\left(\mathbf{H}_{0}\right), \mathbb{R}\right)}\right] \\
& \left.\cdot\left[\sup _{J \in \mathcal{P}_{0}(\mathbb{H})} \int_{0}^{T} \mathbb{E}\left[\left\|\mathbf{B}\left(X_{s}^{J}\right)\right\|_{L\left(U, \mathbf{H}_{\gamma}\right)}^{2}\right] \mathrm{d} s\right]\right) \\
& \cdot\left[\inf _{h \in \mathbb{H} \backslash I}\left|\lambda_{h}\right|\right]^{\beta-\gamma}<\infty \text {. }
\end{aligned}
$$


Proof of Theorem 3.6 Throughout this proof let $\mathbb{U} \subseteq U$ be an orthonormal basis of $U$, let $v^{J}, v_{1,0}^{J}:[0, T] \times \mathbf{P}_{J}\left(\mathbf{H}_{0}\right) \rightarrow \mathbb{R}, J \in \mathcal{P}_{0}(\mathbb{H})$, and $v_{0, \ell}^{J}:[0, T] \times \mathbf{P}_{J}\left(\mathbf{H}_{0}\right) \rightarrow$ $L^{(\ell)}\left(\mathbf{P}_{J}\left(\mathbf{H}_{0}\right), \mathbb{R}\right), \ell \in\{1,2\}, J \in \mathcal{P}_{0}(\mathbb{H})$, be the functions which satisfy for all $J \in$ $\mathcal{P}_{0}(\mathbb{H}),(k, \ell) \in\{(1,0),(0,1),(0,2)\},(t, x) \in[0, T] \times \mathbf{P}_{J}\left(\mathbf{H}_{0}\right)$ that $v^{J}(t, x)=$ $\mathbb{E}\left[\varphi\left(X_{T-t}^{J, x}\right)\right]$ and $v_{k, \ell}^{J}(t, x)=\left(\frac{\partial^{k+\ell}}{\partial t^{k} \partial x^{\ell}} v^{J}\right)(t, x)$, and let $R_{I, J, s}: \Omega \rightarrow L\left(\mathbf{P}_{J}\left(\mathbf{H}_{0}\right)\right)$, $I \in \mathcal{P}(J), J \in \mathcal{P}_{0}(\mathbb{H}), s \in[0, T]$, be the functions which satisfy for all $s \in[0, T]$, $J \in \mathcal{P}_{0}(\mathbb{H}), I \in \mathcal{P}(J), \omega \in \Omega, y_{1}, y_{2} \in \mathbf{P}_{J}\left(\mathbf{H}_{0}\right)$ that

$$
v_{0,2}^{J}\left(s, X_{s}^{I}(\omega)\right)\left(y_{1}, y_{2}\right)=\left\langle y_{1}, R_{I, J, s}(\omega) y_{2}\right\rangle_{\mathbf{H}_{0}} .
$$

Note that for all $J \in \mathcal{P}_{0}(\mathbb{H}),(t, x) \in[0, T] \times \mathbf{P}_{J}\left(\mathbf{H}_{0}\right)$ it holds that $v^{J}(t, x)=$ $u^{J}(T-t, x)$. Next observe that for all $J \in \mathcal{P}_{0}(\mathbb{H}), x \in \mathbf{P}_{J}\left(\mathbf{H}_{0}\right)$ it holds that

$$
\varphi(x)=\mathbb{E}[\varphi(x)]=u^{J}(0, x)=v^{J}(T, x) .
$$

Moreover, note that for all $J \in \mathcal{P}_{0}(\mathbb{H})$ it holds that

$$
\mathbb{E}\left[\varphi\left(X_{T}^{J}\right)\right]=\mathbb{E}\left[u^{J}\left(T, X_{0}^{J}\right)\right]=\mathbb{E}\left[v^{J}\left(0, X_{0}^{J}\right)\right]
$$

Combining (3.18) and (3.19) shows for all $J \in \mathcal{P}_{0}(\mathbb{H}), I \in \mathcal{P}(J)$ that

$$
\begin{aligned}
\mid \mathbb{E} & {\left[\varphi\left(X_{T}^{J}\right)\right]-\mathbb{E}\left[\varphi\left(X_{T}^{I}\right)\right] \mid } \\
& =\left|\mathbb{E}\left[\varphi\left(X_{T}^{I}\right)\right]-\mathbb{E}\left[\varphi\left(X_{T}^{J}\right)\right]\right| \\
& =\left|\mathbb{E}\left[v^{J}\left(T, X_{T}^{I}\right)\right]-\mathbb{E}\left[v^{J}\left(0, X_{0}^{J}\right)\right]\right| \\
& \leq\left|\mathbb{E}\left[v^{J}\left(T, X_{T}^{I}\right)\right]-\mathbb{E}\left[v^{J}\left(0, X_{0}^{I}\right)\right]\right|+\left|\mathbb{E}\left[v^{J}\left(0, X_{0}^{I}\right)\right]-\mathbb{E}\left[v^{J}\left(0, X_{0}^{J}\right)\right]\right| .
\end{aligned}
$$

In a first step we establish an estimate for the second summand on the right hand side of (3.20). For this observe that for all $J \in \mathcal{P}_{0}(\mathbb{H}), I \in \mathcal{P}(J)$ it holds that

$$
\begin{aligned}
\mid \mathbb{E} & {\left[v^{J}\left(0, X_{0}^{I}\right)\right]-\mathbb{E}\left[v^{J}\left(0, X_{0}^{J}\right)\right] \mid } \\
& =\left|\mathbb{E}\left[\int_{0}^{1} v_{0,1}^{J}\left(0, X_{0}^{I}+\tau\left(X_{0}^{J}-X_{0}^{I}\right)\right)\left(X_{0}^{J}-X_{0}^{I}\right) \mathrm{d} \tau\right]\right| \\
& \leq\left|u^{J}(T, \cdot)\right|_{C_{\mathrm{b}}^{1}\left(\mathbf{P}_{J}\left(\mathbf{H}_{0}\right), \mathbb{R}\right)} \mathbb{E}\left[\left\|X_{0}^{J}-X_{0}^{I}\right\|_{\mathbf{H}_{0}}\right] \\
& =\left|u^{J}(T, \cdot)\right|_{C_{\mathrm{b}}\left(\mathbf{P}_{J}\left(\mathbf{H}_{0}\right), \mathbb{R}\right)} \mathbb{E}\left[\left\|\mathbf{P}_{I}\left(X_{0}^{J}\right)-\mathbf{P}_{J}\left(X_{0}^{J}\right)\right\|_{\mathbf{H}_{0}}\right] .
\end{aligned}
$$


In addition, it holds for all $x \in \mathbf{H}_{2(\gamma-\beta)}, I, J \in \mathcal{P}(\mathbb{H})$ with $I \neq J$ that

$$
\begin{aligned}
& \left\|\mathbf{P}_{I}(x)-\mathbf{P}_{J}(x)\right\|_{\mathbf{H}_{0}} \\
& \quad \leq\left\|\boldsymbol{\Lambda}^{2(\beta-\gamma)} \mathbf{P}_{(I \backslash J) \cup(J \backslash I)}\right\|_{L\left(\mathbf{H}_{0}\right)}\left\|\mathbf{P}_{(I \backslash J) \cup(J \backslash I)}(x)\right\|_{\mathbf{H}_{2(\gamma-\beta)}} \\
& \quad=\left[\inf _{h \in(I \backslash J) \cup(J \backslash I)}\left|\lambda_{h}\right|\right]^{\beta-\gamma}\left\|\mathbf{P}_{(I \backslash J) \cup(J \backslash I)}(x)\right\|_{\mathbf{H}_{2(\gamma-\beta)}} \\
& \quad \leq\left[\inf _{h \in(I \backslash J) \cup(J \backslash I)}\left|\lambda_{h}\right|\right]^{\beta-\gamma}\|x\|_{\mathbf{H}_{2(\gamma-\beta)}} .
\end{aligned}
$$

Putting (3.21) and (3.22) together proves for all $J \in \mathcal{P}_{0}(\mathbb{H}), I \in \mathcal{P}(J) \backslash\{\mathbb{H}\}$ that

$$
\begin{aligned}
& \left|\mathbb{E}\left[v^{J}\left(0, X_{0}^{I}\right)\right]-\mathbb{E}\left[v^{J}\left(0, X_{0}^{J}\right)\right]\right| \\
& \leq\left[\sup _{K \in \mathcal{P}_{0}(\mathbb{H})} \sup _{t \in[0, T]}\left|u^{K}(t, \cdot)\right|_{C_{\mathrm{b}}^{1}\left(\mathbf{P}_{K}\left(\mathbf{H}_{0}\right), \mathbb{R}\right)}\right] \mathbb{E}\left[\|\xi\|_{\mathbf{H}_{2(\gamma-\beta)}}\right]\left[\inf _{h \in \mathbb{H} \backslash I}\left|\lambda_{h}\right|\right]^{\beta-\gamma}<\infty .
\end{aligned}
$$

Inequality (3.23) provides an estimate for the second summand on the right hand side of (3.20). In a second step we establish an estimate for the fist summand on the right hand side of (3.20). The chain rule and Lemma 2.2 show that for all $J \in \mathcal{P}_{0}(\mathbb{H})$, $(t, x) \in[0, T] \times \mathbf{P}_{J}\left(\mathbf{H}_{0}\right)$ it holds that

$$
v_{1,0}^{J}(t, x)=-v_{0,1}^{J}(t, x)\left[\mathbf{A} x+\mathbf{P}_{J} \mathbf{F}(x)\right]-\frac{1}{2} \sum_{u \in \mathbb{U}} v_{0,2}^{J}(t, x)\left(\mathbf{P}_{J} \mathbf{B}(x) u, \mathbf{P}_{J} \mathbf{B}(x) u\right)
$$

The standard Itô formula and (3.24) prove for all $J \in \mathcal{P}_{0}(\mathbb{H}), I \in \mathcal{P}(J)$ that

$$
\begin{aligned}
\mathbb{E}\left[v^{J}\left(T, X_{T}^{I}\right)\right]-\mathbb{E}\left[v^{J}\left(0, X_{0}^{I}\right)\right]=\int_{0}^{T} \mathbb{E}\left[v_{1,0}^{J}\left(s, X_{s}^{I}\right)\right] \mathrm{d} s \\
+\int_{0}^{T} \mathbb{E}\left[v_{0,1}^{J}\left(s, X_{s}^{I}\right) \mathbf{A} X_{s}^{I}\right] \mathrm{d} s+\int_{0}^{T} \mathbb{E}\left[v_{0,1}^{J}\left(s, X_{s}^{I}\right) \mathbf{P}_{I} \mathbf{F}\left(X_{s}^{I}\right)\right] \mathrm{d} s \\
+\frac{1}{2} \sum_{b \in \mathbb{U}} \int_{0}^{T} \mathbb{E}\left[v_{0,2}^{J}\left(s, X_{s}^{I}\right)\left(\mathbf{P}_{I} \mathbf{B}\left(X_{s}^{I}\right) b, \mathbf{P}_{I} \mathbf{B}\left(X_{s}^{I}\right) b\right)\right] \mathrm{d} s \\
=\int_{0}^{T} \mathbb{E}\left[v_{0,1}^{J}\left(s, X_{s}^{I}\right) \mathbf{P}_{I} \mathbf{F}\left(X_{s}^{I}\right)\right] \mathrm{d} s-\int_{0}^{T} \mathbb{E}\left[v_{0,1}^{J}\left(s, X_{s}^{I}\right) \mathbf{P}_{J} \mathbf{F}\left(X_{s}^{I}\right)\right] \mathrm{d} s \\
+\frac{1}{2} \sum_{b \in \mathbb{U}} \int_{0}^{T}\left(\mathbb{E}\left[v_{0,2}^{J}\left(s, X_{s}^{I}\right)\left(\mathbf{P}_{I} \mathbf{B}\left(X_{s}^{I}\right) b, \mathbf{P}_{I} \mathbf{B}\left(X_{s}^{I}\right) b\right)\right]\right. \\
\left.\quad-\mathbb{E}\left[v_{0,2}^{J}\left(s, X_{s}^{I}\right)\left(\mathbf{P}_{J} \mathbf{B}\left(X_{s}^{I}\right) b, \mathbf{P}_{J} \mathbf{B}\left(X_{s}^{I}\right) b\right)\right]\right) \mathrm{d} s .
\end{aligned}
$$


This shows for all $J \in \mathcal{P}_{0}(\mathbb{H}), I \in \mathcal{P}(J)$ that

$$
\begin{aligned}
\mid \mathbb{E} & {\left[v^{J}\left(T, X_{T}^{I}\right)\right]-\mathbb{E}\left[v^{J}\left(0, X_{0}^{I}\right)\right]\left|\leq \int_{0}^{T}\right| \mathbb{E}\left[v_{0,1}^{J}\left(s, X_{s}^{I}\right)\left(\mathbf{P}_{I} \mathbf{F}\left(X_{s}^{I}\right)-\mathbf{P}_{J} \mathbf{F}\left(X_{s}^{I}\right)\right)\right] \mid \mathrm{d} s } \\
& +\left|\frac{1}{2} \sum_{b \in \mathbb{U}} \int_{0}^{T} \mathbb{E}\left[v_{0,2}^{J}\left(s, X_{s}^{I}\right)\left(\mathbf{P}_{I} \mathbf{B}\left(X_{s}^{I}\right) b+\mathbf{P}_{J} \mathbf{B}\left(X_{s}^{I}\right) b, \mathbf{P}_{I} \mathbf{B}\left(X_{s}^{I}\right) b-\mathbf{P}_{J} \mathbf{B}\left(X_{s}^{I}\right) b\right)\right] \mathrm{d} s\right|
\end{aligned}
$$

Inequality (3.22) and Lemmas 3.4 and 3.2 thus prove for all $J \in \mathcal{P}_{0}(\mathbb{H}), I \in \mathcal{P}(J) \backslash\{J\}$ that

$$
\begin{aligned}
& \int_{0}^{T}\left|\mathbb{E}\left[v_{0,1}^{J}\left(s, X_{s}^{I}\right)\left(\mathbf{P}_{I} \mathbf{F}\left(X_{s}^{I}\right)-\mathbf{P}_{J} \mathbf{F}\left(X_{s}^{I}\right)\right)\right]\right| \mathrm{d} s \\
& \leq \int_{0}^{T} \mathbb{E}\left[\left|v_{0,1}^{J}\left(s, X_{s}^{I}\right)\left(\mathbf{P}_{I} \mathbf{F}\left(X_{s}^{I}\right)-\mathbf{P}_{J} \mathbf{F}\left(X_{s}^{I}\right)\right)\right|\right] \mathrm{d} s \\
& \leq \sup _{t \in[0, T]}\left|u^{J}(t, \cdot)\right|_{C_{\mathrm{b}}^{1}\left(\mathbf{P}_{J}\left(\mathbf{H}_{0}\right), \mathbb{R}\right)} \int_{0}^{T} \mathbb{E}\left[\left\|\mathbf{P}_{I} \mathbf{F}\left(X_{s}^{I}\right)-\mathbf{P}_{J} \mathbf{F}\left(X_{s}^{I}\right)\right\|_{\mathbf{H}_{0}}\right] \mathrm{d} s \\
& \leq\left[\sup _{K \in \mathcal{P}_{0}(\mathbb{H})} \sup _{t \in[0, T]}\left|u^{K}(t, \cdot)\right|_{C_{\mathrm{b}}^{1}\left(\mathbf{P}_{K}\left(\mathbf{H}_{0}\right), \mathbb{R}\right)}\right] \sup _{K \in \mathcal{P}_{0}(\mathbb{H})} \int_{0}^{T} \mathbb{E}\left[\left\|\mathbf{F}\left(X_{s}^{K}\right)\right\|_{\left.\mathbf{H}_{2(\gamma-\beta)}\right)}\right] \mathrm{d} s \\
& \cdot\left[\inf _{h \in J \backslash I}\left|\lambda_{h}\right|\right]^{\beta-\gamma}<\infty .
\end{aligned}
$$

This estimates the first summand on the right hand side of (3.26). Next we consider the second summand on the right hand side of (3.26). Note that the Hölder inequality for Schatten norms implies for all $s \in[0, T], J \in \mathcal{P}_{0}(\mathbb{H}), I \in \mathcal{P}(J)$ that

$$
\begin{aligned}
& \left\|\mathbf{B}\left(X_{s}^{I}\right)^{\star}\left(\mathbf{P}_{I}+\mathbf{P}_{J}\right) R_{I, J, s}\left(\mathbf{P}_{I}-\mathbf{P}_{J}\right) \mathbf{B}\left(X_{s}^{I}\right)\right\|_{L_{1}(U)} \\
& \leq\left\|\mathbf{B}\left(X_{s}^{I}\right)^{\star}\left(\mathbf{P}_{I}+\mathbf{P}_{J}\right)\right\|_{L_{(2 \beta) / \gamma}\left(\mathbf{H}_{0}, U\right)}\left\|R_{I, J, s}\right\|_{L\left(\mathbf{P}_{J}\left(\mathbf{H}_{0}\right)\right)} \\
& \quad \cdot\left\|\left(\mathbf{P}_{I}-\mathbf{P}_{J}\right) \mathbf{B}\left(X_{s}^{I}\right)\right\|_{L_{(2 \beta) /(2 \beta-\gamma)}\left(U, \mathbf{H}_{0}\right)} .
\end{aligned}
$$

Moreover, observe that for all $s \in[0, T], J \in \mathcal{P}_{0}(\mathbb{H}), I \in \mathcal{P}(J) \backslash\{J\}$ it holds that

$$
\begin{aligned}
& \left\|\mathbf{B}\left(X_{S}^{I}\right)^{\star}\left(\mathbf{P}_{I}+\mathbf{P}_{J}\right)\right\|_{L_{(2 \beta) / \gamma}\left(\mathbf{H}_{0}, U\right)}=\left\|\mathbf{B}\left(X_{s}^{I}\right)^{\star} \boldsymbol{\Lambda}^{\gamma} \boldsymbol{\Lambda}^{-\gamma}\left(\mathbf{P}_{I}+\mathbf{P}_{J}\right)\right\|_{L_{(2 \beta) / \gamma}\left(\mathbf{H}_{0}, U\right)} \\
& \leq\left\|\mathbf{B}\left(X_{s}^{I}\right)^{\star} \boldsymbol{\Lambda}^{\gamma}\right\|_{L\left(\mathbf{H}_{0}, U\right)}\left\|\boldsymbol{\Lambda}^{-\gamma}\right\|_{L_{(2 \beta) / \gamma}\left(\mathbf{H}_{0}\right)}\left\|\mathbf{P}_{I}+\mathbf{P}_{J}\right\|_{L\left(\mathbf{H}_{0}\right)} \\
& =\left\|\mathbf{B}\left(X_{s}^{I}\right)\right\|_{L\left(U, \mathbf{H}_{\gamma}\right)}\left\|\boldsymbol{\Lambda}^{-\beta}\right\|_{L_{2}\left(\mathbf{H}_{0}\right)}^{\gamma / \beta}\left\|\mathbf{P}_{I}+\mathbf{P}_{J}\right\|_{L\left(\mathbf{H}_{0}\right)} \\
& \leq 2\left\|\mathbf{B}\left(X_{s}^{I}\right)\right\|_{L\left(U, \mathbf{H}_{\gamma}\right)}\left\|\boldsymbol{\Lambda}^{-\beta}\right\|_{L_{2}\left(\mathbf{H}_{0}\right)}^{\gamma / \beta}<\infty
\end{aligned}
$$

and

$$
\begin{aligned}
& \left\|\left(\mathbf{P}_{I}-\mathbf{P}_{J}\right) \mathbf{B}\left(X_{s}^{I}\right)\right\|_{L_{(2 \beta) /(2 \beta-\gamma)}\left(U, \mathbf{H}_{0}\right)} \\
& \leq\left\|\left.\left(\mathbf{P}_{I}-\mathbf{P}_{J}\right)\right|_{\mathbf{H}_{\gamma}}\right\|_{L_{(2 \beta) /(2 \beta-\gamma)}\left(\mathbf{H}_{\gamma}, \mathbf{H}_{0}\right)}\left\|\mathbf{B}\left(X_{s}^{I}\right)\right\|_{L\left(U, \mathbf{H}_{\gamma}\right)}
\end{aligned}
$$




$$
\begin{aligned}
& \leq\left\|\left(\mathbf{P}_{I}-\mathbf{P}_{J}\right) \boldsymbol{\Lambda}^{2(\beta-\gamma)}\right\|_{L\left(\mathbf{H}_{0}\right)}\left\|\boldsymbol{\Lambda}^{2(\gamma-\beta)} \mid \mathbf{H}_{\gamma}\right\|_{L_{(2 \beta) /(2 \beta-\gamma)}\left(\mathbf{H}_{\gamma}, \mathbf{H}_{0}\right)}\left\|\mathbf{B}\left(X_{s}^{I}\right)\right\|_{L\left(U, \mathbf{H}_{\gamma}\right)} \\
& =\left[\inf _{h \in J \backslash I}\left|\lambda_{h}\right|\right]^{\beta-\gamma}\left\|\boldsymbol{\Lambda}^{-\beta}\right\|_{L_{2}\left(\mathbf{H}_{0}\right) / \beta}^{(2 \beta-\gamma)}\left\|\mathbf{B}\left(X_{S}^{I}\right)\right\|_{L\left(U, \mathbf{H}_{\gamma}\right)}<\infty .
\end{aligned}
$$

In addition, Lemma 3.4 establishes for all $s \in[0, T], J \in \mathcal{P}_{0}(\mathbb{H}), I \in \mathcal{P}(J)$ that

$$
\left\|R_{I, J, S}\right\|_{L\left(\mathbf{P}_{J}\left(\mathbf{H}_{0}\right)\right)} \leq \sup _{t \in[0, T]}\left|u^{J}(t, \cdot)\right|_{C_{\mathrm{b}}^{2}\left(\mathbf{P}_{J}\left(\mathbf{H}_{0}\right), \mathbb{R}\right)}<\infty
$$

Combining (3.28)-(3.31) shows for all $s \in[0, T], J \in \mathcal{P}_{0}(\mathbb{H}), I \in \mathcal{P}(J) \backslash\{J\}$ that

$$
\begin{gathered}
\left\|\mathbf{B}\left(X_{s}^{I}\right)^{\star}\left(\mathbf{P}_{I}+\mathbf{P}_{J}\right) R_{I, J, s}\left(\mathbf{P}_{I}-\mathbf{P}_{J}\right) \mathbf{B}\left(X_{s}^{I}\right)\right\|_{L_{1}(U)} \\
\leq 2\left\|\boldsymbol{\Lambda}^{-\beta}\right\|_{L_{2}\left(\mathbf{H}_{0}\right)}^{2}\left[\sup _{t \in[0, T]}\left|u^{J}(t, \cdot)\right|_{C_{\mathrm{b}}^{2}\left(\mathbf{P}_{J}\left(\mathbf{H}_{0}\right), \mathbb{R}\right)}\right] \\
\cdot\left\|\mathbf{B}\left(X_{s}^{I}\right)\right\|_{L\left(U, \mathbf{H}_{\gamma}\right)}^{2}\left[\inf _{h \in J \backslash I}\left|\lambda_{h}\right|\right]^{\beta-\gamma}<\infty .
\end{gathered}
$$

This and (3.17) imply for all $s \in[0, T], J \in \mathcal{P}_{0}(\mathbb{H}), I \in \mathcal{P}(J) \backslash\{J\}$ that

$$
\begin{aligned}
& \left|\sum_{b \in \mathbb{U}} \mathbb{E}\left[v_{0,2}^{J}\left(s, X_{s}^{I}\right)\left(\left(\mathbf{P}_{I}+\mathbf{P}_{J}\right) \mathbf{B}\left(X_{s}^{I}\right) b,\left(\mathbf{P}_{I}-\mathbf{P}_{J}\right) \mathbf{B}\left(X_{s}^{I}\right) b\right)\right]\right| \\
& =\left|\mathbb{E}\left[\sum_{b \in \mathbb{U}}\left\langle\left(\mathbf{P}_{I}+\mathbf{P}_{J}\right) \mathbf{B}\left(X_{s}^{I}\right) b, R_{I, J, s}\left(\mathbf{P}_{I}-\mathbf{P}_{J}\right) \mathbf{B}\left(X_{s}^{I}\right) b\right\rangle_{\mathbf{H}_{0}}\right]\right| \\
& =\left|\mathbb{E}\left[\sum_{b \in \mathbb{U}}\left\langle b, \mathbf{B}\left(X_{s}^{I}\right)^{\star}\left(\mathbf{P}_{I}+\mathbf{P}_{J}\right) R_{I, J, s}\left(\mathbf{P}_{I}-\mathbf{P}_{J}\right) \mathbf{B}\left(X_{s}^{I}\right) b\right\rangle_{U}\right]\right| \\
& =\left|\mathbb{E}\left[\operatorname{trace}{ }_{U}\left(\mathbf{B}\left(X_{s}^{I}\right)^{\star}\left(\mathbf{P}_{I}+\mathbf{P}_{J}\right) R_{I, J, s}\left(\mathbf{P}_{I}-\mathbf{P}_{J}\right) \mathbf{B}\left(X_{s}^{I}\right)\right)\right]\right| \\
& \leq \mathbb{E}\left[\left\|\mathbf{B}\left(X_{s}^{I}\right)^{\star}\left(\mathbf{P}_{I}+\mathbf{P}_{J}\right) R_{I, J, s}\left(\mathbf{P}_{I}-\mathbf{P}_{J}\right) \mathbf{B}\left(X_{s}^{I}\right)\right\|_{L_{1}(U)}\right] \\
& \leq 2\left\|\mathbf{\Lambda}^{-\beta}\right\|_{L_{2}\left(\mathbf{H}_{0}\right)}^{2}\left[\sup _{t \in[0, T]}\left|u^{J}(t, \cdot)\right|_{C_{\mathrm{b}}^{2}\left(\mathbf{P}_{J}\left(\mathbf{H}_{0}\right), \mathbb{R}\right)}\right] \mathbb{E}\left[\left\|\mathbf{B}\left(X_{s}^{I}\right)\right\|_{L\left(U, \mathbf{H}_{\gamma}\right)}^{2}\right]\left[\inf _{h \in J \backslash I}\left|\lambda_{h}\right|\right]^{\beta-\gamma} .
\end{aligned}
$$

Lemmas 3.2 and 3.4 hence prove for all $J \in \mathcal{P}_{0}(\mathbb{H}), I \in \mathcal{P}(J) \backslash\{J\}$ that

$$
\begin{aligned}
\mid & \frac{1}{2} \sum_{b \in \mathbb{U}} \int_{0}^{T} \mathbb{E}\left[v_{0,2}^{J}\left(s, X_{s}^{I}\right)\left(\mathbf{P}_{I} \mathbf{B}\left(X_{s}^{I}\right) b+\mathbf{P}_{J} \mathbf{B}\left(X_{s}^{I}\right) b, \mathbf{P}_{I} \mathbf{B}\left(X_{s}^{I}\right) b-\mathbf{P}_{J} \mathbf{B}\left(X_{s}^{I}\right) b\right)\right] \mathrm{d} s \mid \\
\leq & \left\|\mathbf{\Lambda}^{-\beta}\right\|_{L_{2}\left(\mathbf{H}_{0}\right)}^{2}\left[\sup _{K \in \mathcal{P}_{0}(\mathbb{H})} \sup _{t \in[0, T]}\left|u^{K}(t, \cdot)\right|_{C_{\mathrm{b}}^{2}\left(\mathbf{P}_{K}\left(\mathbf{H}_{0}\right), \mathbb{R}\right)}\right] \\
& \cdot\left[\sup _{K \in \mathcal{P}_{0}(\mathbb{H})} \int_{0}^{T} \mathbb{E}\left[\left\|\mathbf{B}\left(X_{s}^{K}\right)\right\|_{L\left(U, \mathbf{H}_{\gamma}\right)}^{2}\right] \mathrm{d} s\right]\left[\inf _{h \in J \backslash I}\left|\lambda_{h}\right|\right]^{\beta-\gamma}<\infty
\end{aligned}
$$


Combining this with (3.26) and (3.27) ensures for all $J \in \mathcal{P}_{0}(\mathbb{H}), I \in \mathcal{P}(J) \backslash\{\mathbb{H}\}$ that

$$
\begin{aligned}
\mid \mathbb{E} & {\left[v^{J}\left(T, X_{T}^{I}\right)\right]-\mathbb{E}\left[v^{J}\left(0, X_{0}^{I}\right)\right] \mid } \\
\leq & \left(\left[\sup _{K \in \mathcal{P}_{0}(\mathbb{H})} \sup _{t \in[0, T]}\left|u^{K}(t, \cdot)\right|_{\left.C_{\mathrm{b}}^{1}\left(\mathbf{P}_{K}\left(\mathbf{H}_{0}\right), \mathbb{R}\right)\right]} \sup _{K \in \mathcal{P}_{0}(\mathbb{H})} \int_{0}^{T} \mathbb{E}\left[\left\|\mathbf{F}\left(X_{s}^{K}\right)\right\|_{\mathbf{H}_{2(\gamma-\beta)}}\right] \mathrm{d} s\right.\right. \\
& +\left\|\boldsymbol{\Lambda}^{-\beta}\right\|_{L_{2}\left(\mathbf{H}_{0}\right)}^{2}\left[\sup _{K \in \mathcal{P}_{0}(\mathbb{H})} \sup _{t \in[0, T]}\left|u^{K}(t, \cdot)\right|_{C_{\mathrm{b}}^{2}\left(\mathbf{P}_{K}\left(\mathbf{H}_{0}\right), \mathbb{R}\right)}\right] \\
& \left.\cdot\left[\sup _{K \in \mathcal{P}_{0}(\mathbb{H})} \int_{0}^{T} \mathbb{E}\left[\left\|\mathbf{B}\left(X_{s}^{K}\right)\right\|_{L\left(U, \mathbf{H}_{\gamma}\right)}^{2}\right] \mathrm{d} s\right]\right)\left[\inf _{h \in \mathbb{H} \backslash I}\left|\lambda_{h}\right|\right]^{\beta-\gamma}<\infty
\end{aligned}
$$

This constitutes an estimate for the first summand on the right hand side of (3.20). Inequalities (3.35), (3.20), and (3.23) show for all $J \in \mathcal{P}_{0}(\mathbb{H}), I \in \mathcal{P}(J) \backslash\{\mathbb{H}\}$ that

$$
\begin{aligned}
\mid \mathbb{E} & {\left[\varphi\left(X_{T}^{J}\right)\right]-\mathbb{E}\left[\varphi\left(X_{T}^{I}\right)\right] \mid } \\
\leq & \left([ \operatorname { s u p } _ { K \in \mathcal { P } _ { 0 } ( \mathbb { H } ) } \operatorname { s u p } _ { t \in [ 0 , T ] } | u ^ { K } ( t , \cdot ) | _ { C _ { \mathrm { b } } ^ { 1 } ( \mathbf { P } _ { K } ( \mathbf { H } _ { 0 } ) , \mathbb { R } ) } ] \left[\mathbb{E}\left[\|\xi\|_{\left.\mathbf{H}_{2(\gamma-\beta)}\right]}\right]\right.\right. \\
& \left.+\sup _{K \in \mathcal{P}_{0}(\mathbb{H})} \int_{0}^{T} \mathbb{E}\left[\left\|\mathbf{F}\left(X_{s}^{K}\right)\right\|_{\mathbf{H}_{2(\gamma-\beta)}}\right] \mathrm{d} s\right] \\
& +\left\|\mathbf{\Lambda}^{-\beta}\right\|_{L_{2}\left(\mathbf{H}_{0}\right)}^{2}\left[\sup _{K \in \mathcal{P}_{0}(\mathbb{H})} \sup _{t \in[0, T]}\left|u^{K}(t, \cdot)\right|_{C_{\mathrm{b}}^{2}\left(\mathbf{P}_{K}\left(\mathbf{H}_{0}\right), \mathbb{R}\right)}\right] \\
& \left.\cdot\left[\sup _{K \in \mathcal{P}_{0}(\mathbb{H})} \int_{0}^{T} \mathbb{E}\left[\left\|\mathbf{B}\left(X_{s}^{K}\right)\right\|_{L\left(U, \mathbf{H}_{\gamma}\right)}^{2}\right] \mathrm{d} s\right]\right)\left[\inf _{h \in \mathbb{H} \backslash I}\left|\lambda_{h}\right|\right]^{\beta-\gamma}<\infty .
\end{aligned}
$$

In a third step Lemmas 3.3, 2.5, and 2.6, the Cauchy-Schwarz inequality, and the Burkholder-Davis-Gundy inequality (see, e.g., Lemma 7.7 in Da Prato \& Zabczyk [13]) imply for all $n \in \mathbb{N},\left(J_{k}\right)_{k \in \mathbb{N}_{0}} \subseteq \mathcal{P}(\mathbb{H})$ with $\bigcup_{k=1}^{\infty} J_{k}=J_{0}$ and $\forall k \in \mathbb{N}: J_{k} \subseteq$ $J_{k+1} \in \mathcal{P}_{0}(\mathbb{H})$ that

$$
\begin{aligned}
\sup _{t \in[0, T]}\left(\mathbb{E}\left[\left\|X_{t}^{J_{0}}-X_{t}^{J_{n}}\right\|_{\mathbf{H}_{0}}^{2}\right]\right)^{1 / 2} \\
\leq \sqrt{2}\left[\sup _{t \in[0, T]}\left(\mathbb{E}\left[\left\|\mathbf{P}_{J_{0} \backslash J_{n}} X_{t}^{J_{0}}\right\|_{\mathbf{H}_{0}}^{2}\right]\right)^{1 / 2}\right] \\
\cdot \exp \left(\frac{1}{2}\left[\sqrt{2} T\left|\mathbf{P}_{J_{n}} \mathbf{F}\right|_{\operatorname{Lip}^{0}\left(\mathbf{H}_{0}, \mathbf{H}_{0}\right)}+\sqrt{2 T}\left|\mathbf{P}_{J_{n}} \mathbf{B}\right|_{\operatorname{Lip}^{0}\left(\mathbf{H}_{0}, L_{2}\left(U, \mathbf{H}_{0}\right)\right)}\right]^{2}\right) \\
\leq \sqrt{2} \exp \left(\frac{1}{2}\left[\sqrt{2} T|\mathbf{F}|_{\operatorname{Lip}^{0}\left(\mathbf{H}_{0}, \mathbf{H}_{0}\right)}+\sqrt{2 T}|\mathbf{B}|_{\operatorname{Lip}^{0}\left(\mathbf{H}_{0}, L_{2}\left(U, \mathbf{H}_{0}\right)\right)}\right]^{2}\right) \\
\cdot\left(\left(\mathbb{E}\left[\left\|\mathbf{P}_{J_{0} \backslash J_{n}} \xi\right\|_{\mathbf{H}_{0}}^{2}\right]\right)^{1 / 2}+\left[T \int_{0}^{T} \mathbb{E}\left[\left\|\mathbf{P}_{J_{0} \backslash J_{n}} \mathbf{F}\left(X_{S}^{J_{0}}\right)\right\|_{\mathbf{H}_{0}}^{2}\right] \mathrm{d} s\right]^{1 / 2}\right. \\
\left.+\left[\int_{0}^{T} \mathbb{E}\left[\left\|\mathbf{P}_{J_{0} \backslash J_{n}} \mathbf{B}\left(X_{s}^{J_{0}}\right)\right\|_{L_{2}\left(U, \mathbf{H}_{0}\right)}^{2}\right] \mathrm{d} s\right]^{1 / 2}\right) .
\end{aligned}
$$


Therefore, Lebesgue's theorem of dominated convergence proves for all $\left(J_{k}\right)_{k \in \mathbb{N}_{0}} \subseteq$ $\mathcal{P}(\mathbb{H})$ with $\bigcup_{k=1}^{\infty} J_{k}=J_{0}$ and $\forall k \in \mathbb{N}: J_{k} \subseteq J_{k+1} \in \mathcal{P}_{0}(\mathbb{H})$ that

$$
\limsup _{n \rightarrow \infty} \sup _{t \in[0, T]}\left(\mathbb{E}\left[\left\|X_{t}^{J_{0}}-X_{t}^{J_{n}}\right\|_{\mathbf{H}_{0}}^{2}\right]\right)^{1 / 2}=0 .
$$

Moreover, observe that (3.36) ensures for all $n \in \mathbb{N}, I \in \mathcal{P}_{0}(\mathbb{H}) \backslash\{\mathbb{H}\},\left(J_{k}\right)_{k \in \mathbb{N}} \subseteq$ $\mathcal{P}_{0}(\mathbb{H})$ with $\bigcup_{k=1}^{\infty} J_{k}=\mathbb{H}$ and $\forall k \in \mathbb{N}: I \subseteq J_{k} \subseteq J_{k+1}$ that

$$
\begin{aligned}
\mid \mathbb{E} & {\left[\varphi\left(X_{T}^{\mathbb{H}}\right)\right]-\mathbb{E}\left[\varphi\left(X_{T}^{I}\right)\right] \mid } \\
\leq & \left|\mathbb{E}\left[\varphi\left(X_{T}^{\mathbb{H}}\right)\right]-\mathbb{E}\left[\varphi\left(X_{T}^{J_{n}}\right)\right]\right|+\left|\mathbb{E}\left[\varphi\left(X_{T}^{J_{n}}\right)\right]-\mathbb{E}\left[\varphi\left(X_{T}^{I}\right)\right]\right| \\
\leq & |\varphi|_{C_{\mathrm{b}}^{1}\left(\mathbf{H}_{0}, \mathbb{R}\right)}\left(\mathbb{E}\left[\left\|X_{T}^{\mathbb{H}}-X_{T}^{J_{n}}\right\|_{\mathbf{H}_{0}}^{2}\right]\right)^{1 / 2} \\
& +\left([ \operatorname { s u p } _ { K \in \mathcal { P } _ { 0 } ( \mathbb { H } ) } \operatorname { s u p } _ { t \in [ 0 , T ] } | u ^ { K } ( t , \cdot ) | _ { C _ { \mathrm { b } } ^ { 1 } ( \mathbf { P } _ { K } ( \mathbf { H } _ { 0 } ) , \mathbb { R } ) } ] \left[\mathbb{E}\left[\|\xi\|_{\mathbf{H}_{2(\gamma-\beta)}}\right]\right.\right. \\
& \left.+\sup _{K \in \mathcal{P}_{0}(\mathbb{H})} \int_{0}^{T} \mathbb{E}\left[\left\|\mathbf{F}\left(X_{S}^{K}\right)\right\|_{\mathbf{H}_{2(\gamma-\beta)}}\right] \mathrm{d} s\right] \\
& +\left\|\mathbf{\Lambda}^{-\beta}\right\|_{L_{2}\left(\mathbf{H}_{0}\right)}^{2}\left[\sup _{K \in \mathcal{P}_{0}(\mathbb{H})} \sup _{t \in[0, T]}\left|u^{K}(t, \cdot)\right|_{C_{\mathrm{b}}^{2}\left(\mathbf{P}_{K}\left(\mathbf{H}_{0}\right), \mathbb{R}\right)}\right] \\
& \left.\cdot\left[\sup _{K \in \mathcal{P}_{0}(\mathbb{H})} \int_{0}^{T} \mathbb{E}\left[\left\|\mathbf{B}_{0}^{K}\left(X_{S}^{K}\right)\right\|_{L\left(U, \mathbf{H}_{\gamma}\right)}^{2}\right] \mathrm{d} s\right]\right)\left[\inf _{h \in \mathbb{H} \backslash I}\left|\lambda_{h}\right|\right]^{\beta-\gamma} .
\end{aligned}
$$

Note that (3.38) and letting $n \rightarrow \infty$ in (3.39) complete the proof of Theorem 3.6 in the case that $I \in \mathcal{P}_{0}(\mathbb{H}) \backslash\{\mathbb{H}\}$. In a last step we prove the remaining cases. Estimate (3.39) ensures for all $n \in \mathbb{N}, I_{0} \in \mathcal{P}(\mathbb{H}) \backslash\{\mathbb{H}\},\left(I_{k}\right)_{k \in \mathbb{N}} \subseteq \mathcal{P}_{0}\left(I_{0}\right)$ with $\bigcup_{k=1}^{\infty} I_{k}=I_{0}$ and $\forall k \in \mathbb{N}: I_{k} \subseteq I_{k+1}$ that

$$
\begin{aligned}
& \left|\mathbb{E}\left[\varphi\left(X_{T}^{\mathbb{H}}\right)\right]-\mathbb{E}\left[\varphi\left(X_{T}^{I_{0}}\right)\right]\right| \\
& \leq\left|\mathbb{E}\left[\varphi\left(X_{T}^{\mathbb{H}}\right)\right]-\mathbb{E}\left[\varphi\left(X_{T}^{I_{n}}\right)\right]\right|+\left|\mathbb{E}\left[\varphi\left(X_{T}^{I_{0}}\right)\right]-\mathbb{E}\left[\varphi\left(X_{T}^{I_{n}}\right)\right]\right| \\
& \leq\left([ \operatorname { s u p } _ { J \in \mathcal { P } _ { 0 } ( \mathbb { H } ) } \operatorname { s u p } _ { t \in [ 0 , T ] } | u ^ { J } ( t , \cdot ) | _ { C _ { \mathrm { b } } ^ { 1 } ( \mathbf { P } _ { J } ( \mathbf { H } _ { 0 } ) , \mathbb { R } ) } ] \left[\mathbb{E}\left[\|\xi\|_{\mathbf{H}_{2(\gamma-\beta)}}\right]\right.\right. \\
& \left.+\sup _{J \in \mathcal{P}_{0}(\mathbb{H})} \int_{0}^{T} \mathbb{E}\left[\left\|\mathbf{F}\left(X_{s}^{J}\right)\right\|_{\mathbf{H}_{2(\gamma-\beta)}}\right] \mathrm{d} s\right] \\
& +\left\|\boldsymbol{\Lambda}^{-\beta}\right\|_{L_{2}\left(\mathbf{H}_{0}\right)}^{2}\left[\sup _{J \in \mathcal{P}_{0}(\mathbb{H})} \sup _{t \in[0, T]}\left|u^{J}(t, \cdot)\right|_{C_{\mathrm{b}}^{2}\left(\mathbf{P}_{J}\left(\mathbf{H}_{0}\right), \mathbb{R}\right)}\right] \\
& \left.\cdot\left[\sup _{J \in \mathcal{P}_{0}(\mathbb{H})} \int_{0}^{T} \mathbb{E}\left[\left\|\mathbf{B}\left(X_{s}^{J}\right)\right\|_{L\left(U, \mathbf{H}_{\gamma}\right)}^{2}\right] \mathrm{d} s\right]\right)\left[\inf _{h \in \mathbb{H} \backslash I}\left|\lambda_{h}\right|\right]^{\beta-\gamma} \\
& +|\varphi|_{C_{\mathrm{b}}^{1}\left(\mathbf{H}_{0}, \mathbb{R}\right)}\left(\mathbb{E}\left[\left\|X_{T}^{I_{0}}-X_{T}^{I_{n}}\right\|_{\mathbf{H}_{0}}^{2}\right]\right)^{1 / 2} .
\end{aligned}
$$

Equation (3.38) and Lemma 3.5 thus complete the proof of Theorem 3.6. 
The next corollary is a direct consequence of Theorem 3.6 and Lemma 3.2.

Corollary 3.7 Assume the setting in Sect. 3.1, let $X^{I}:[0, T] \times \Omega \rightarrow \mathbf{P}_{I}\left(\mathbf{H}_{\rho}\right), I \in$ $\mathcal{P}(\mathbb{H})$, and $X^{J, x}:[0, T] \times \Omega \rightarrow \mathbf{P}_{J}\left(\mathbf{H}_{0}\right), x \in \mathbf{P}_{J}\left(\mathbf{H}_{0}\right), J \in \mathcal{P}_{0}(\mathbb{H})$, be $\left(\mathbb{F}_{t}\right)_{t \in[0, T]^{-}}$ predictable stochastic processes such that for all $I \in \mathcal{P}(\mathbb{H}), J \in \mathcal{P}_{0}(\mathbb{H}), x \in \mathbf{P}_{J}\left(\mathbf{H}_{0}\right)$, $t \in[0, T]$ it holds that $\sup _{s \in[0, T]} \mathbb{E}\left[\left\|X_{s}^{I}\right\|_{\mathbf{H}_{\rho}}^{2}+\left\|X_{s}^{J, x}\right\|_{\mathbf{H}_{0}}^{2}\right]<\infty$ and $\mathbb{P}$-a.s. that

$$
\begin{aligned}
X_{t}^{I} & =\mathrm{e}^{t \mathbf{A}} \mathbf{P}_{I} \xi+\int_{0}^{t} \mathrm{e}^{(t-s) \mathbf{A}} \mathbf{P}_{I} \mathbf{F}\left(X_{s}^{I}\right) \mathrm{d} s+\int_{0}^{t} \mathrm{e}^{(t-s) \mathbf{A}} \mathbf{P}_{I} \mathbf{B}\left(X_{s}^{I}\right) \mathrm{d} W_{s}, \\
X_{t}^{J, x} & =\mathrm{e}^{t \mathbf{A}} x+\int_{0}^{t} \mathrm{e}^{(t-s) \mathbf{A}} \mathbf{P}_{J} \mathbf{F}\left(X_{s}^{J, x}\right) \mathrm{d} s+\int_{0}^{t} \mathrm{e}^{(t-s) \mathbf{A}} \mathbf{P}_{J} \mathbf{B}\left(X_{s}^{J, x}\right) \mathrm{d} W_{s},
\end{aligned}
$$

let $\varphi \in C_{\mathrm{b}}^{2}\left(\mathbf{H}_{0}, \mathbb{R}\right)$, and let $u^{J}:[0, T] \times \mathbf{P}_{J}\left(\mathbf{H}_{0}\right) \rightarrow \mathbb{R}, J \in \mathcal{P}_{0}(\mathbb{H})$, be the functions which satisfyfor all $J \in \mathcal{P}_{0}(\mathbb{H}),(t, x) \in[0, T] \times \mathbf{P}_{J}\left(\mathbf{H}_{0}\right)$ that $u^{J}(t, x)=\mathbb{E}\left[\varphi\left(X_{t}^{J, x}\right)\right]$. Then it holds for all $I \in \mathcal{P}(\mathbb{H}) \backslash\{\mathbb{H}\}$ that

$$
\begin{aligned}
&\left|\mathbb{E}\left[\varphi\left(X_{T}^{\mathbb{H}}\right)\right]-\mathbb{E}\left[\varphi\left(X_{T}^{I}\right)\right]\right| \\
& \leq\left[\max _{i \in\{1,2\}} \sup _{J \in \mathcal{P}_{0}(\mathbb{H})} \sup _{t \in[0, T]}\left|u^{J}(t, \cdot)\right|_{C_{\mathrm{b}}^{i}\left(\mathbf{P}_{J}\left(\mathbf{H}_{0}\right), \mathbb{R}\right)}\right] \sup _{J \in \mathcal{P}_{0}(\mathbb{H})} \sup _{t \in[0, T]} \max \left\{1, \mathbb{E}\left[\left\|X_{t}^{J}\right\|_{\mathbf{H}_{\rho}}^{2}\right]\right\} \\
& \cdot\left(\mathbb{E}\left[\|\xi\|_{\mathbf{H}_{2(\gamma-\beta)}}\right]+T\left\|\left.\mathbf{F}\right|_{\mathbf{H}_{\rho}}\right\|_{\operatorname{Lip}^{0}\left(\mathbf{H}_{\rho}, \mathbf{H}_{2(\gamma-\beta)}\right)}\right. \\
&\left.+T\left\|\boldsymbol{\Lambda}^{-\beta}\right\|_{L_{2}\left(\mathbf{H}_{0}\right)}^{2}\left\|\left.\mathbf{B}\right|_{\mathbf{H}_{\rho}}\right\|_{\operatorname{Lip}^{0}\left(\mathbf{H}_{\rho}, L\left(U, \mathbf{H}_{\gamma}\right)\right)}^{2}\right) \\
& \cdot\left[\inf _{h \in \mathbb{H} \backslash I}\left|\lambda_{h}\right|\right]^{\beta-\gamma}<\infty .
\end{aligned}
$$

The last result in this section, Corollary 3.8 below, follows immediately from Corollary 3.7 and Lemmas 3.4 and 3.2.

Corollary 3.8 Assume the setting in Sect. 3.1 and let $X^{I}:[0, T] \times \Omega \rightarrow \mathbf{P}_{I}\left(\mathbf{H}_{\rho}\right)$,

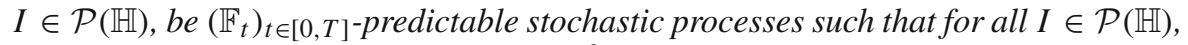
$t \in[0, T]$ it holds that $\sup _{s \in[0, T]} \mathbb{E}\left[\left\|X_{s}^{I}\right\|_{\mathbf{H}_{\rho}}^{2}\right]<\infty$ and $\mathbb{P}$-a.s. that

$$
X_{t}^{I}=\mathrm{e}^{t \mathbf{A}} \mathbf{P}_{I} \xi+\int_{0}^{t} \mathrm{e}^{(t-s) \mathbf{A}} \mathbf{P}_{I} \mathbf{F}\left(X_{s}^{I}\right) \mathrm{d} s+\int_{0}^{t} \mathrm{e}^{(t-s) \mathbf{A}} \mathbf{P}_{I} \mathbf{B}\left(X_{s}^{I}\right) \mathrm{d} W_{s} .
$$


Then it holds for all $\varphi \in C_{\mathrm{b}}^{2}\left(\mathbf{H}_{0}, \mathbb{R}\right), I \in \mathcal{P}(\mathbb{H}) \backslash\{\mathbb{H}\}$ that

$$
\begin{aligned}
\mid \mathbb{E}[ & \left.\varphi\left(X_{T}^{\mathbb{H}}\right)\right]-\mathbb{E}\left[\varphi\left(X_{T}^{I}\right)\right] \mid \\
\leq & \|\varphi\|_{C_{\mathrm{b}}^{2}\left(\mathbf{H}_{0}, \mathbb{R}\right)} \max \left\{1, \mathbb{E}\left[\|\xi\|_{\mathbf{H}_{\rho}}^{2}\right]\right\} \\
& \cdot\left(\mathbb{E}\left[\|\xi\|_{\mathbf{H}_{2(\gamma-\beta)}}\right]+T\left\|\left.\mathbf{F}\right|_{\mathbf{H}_{\rho}}\right\|_{\operatorname{Lip}^{0}\left(\mathbf{H}_{\rho}, \mathbf{H}_{2(\gamma-\beta)}\right)}\right. \\
& \left.\quad+T\left\|\boldsymbol{\Lambda}^{-\beta}\right\|_{L_{2}\left(\mathbf{H}_{0}\right)}^{2}\left\|\left.\mathbf{B}\right|_{\mathbf{H}_{\rho}}\right\|_{\operatorname{Lip}^{0}\left(\mathbf{H}_{\rho}, L\left(U, \mathbf{H}_{\gamma}\right)\right)}^{2}\right) \\
& \cdot \max \left\{1,\left[T\left(\left(C_{\mathbf{F})^{2}}+2\left(C_{\mathbf{B}}\right)^{2}\right)\right]^{1 / 2}\right\}\right. \\
& \cdot \exp \left(T\left[\frac{1}{2}+3|\mathbf{F}|_{\operatorname{Lip}^{0}\left(\mathbf{H}_{0}, \mathbf{H}_{0}\right)}+4|\mathbf{B}|_{\operatorname{Lip}^{0}\left(\mathbf{H}_{0}, L_{2}\left(U, \mathbf{H}_{0}\right)\right)}^{2}\right]\right) \\
& \cdot \exp \left(T\left[2\left\|\left.\mathbf{F}\right|_{\mathbf{H}_{\rho}}\right\|_{\operatorname{Lip}^{0}\left(\mathbf{H}_{\rho}, \mathbf{H}_{\rho}\right)}+\left\|\left.\mathbf{B}\right|_{\mathbf{H}_{\rho}}\right\|_{\operatorname{Lip}^{0}\left(\mathbf{H}_{\rho}, L_{2}\left(U, \mathbf{H}_{\rho}\right)\right)}^{2}\right]\right) \\
& \cdot\left[\inf _{h \in \mathbb{H} \backslash I}\left|\lambda_{h}\right|\right]^{\beta-\gamma}<\infty .
\end{aligned}
$$

\subsection{Semilinear Stochastic Wave Equations and the Continuous Version of the Hyperbolic Anderson Model}

Roughly speaking, the following elementary and well-known lemma provides a useful criterion for determining whether a vector belonging to an interpolation space associated to a symmetric diagonal linear operator possesses more regularity (cf., e.g., Example 37.1 in Sell \& You [40]).

Lemma 3.9 Consider the notation in Sect. 1.1 , let $\mathbb{K} \in\{\mathbb{R}, \mathbb{C}\}$, let $\left(H,\langle\cdot, \cdot\rangle_{H},\|\cdot\|_{H}\right)$ be a $\mathbb{K}$-Hilbert space, let $\mathbb{H} \subseteq H$ be a nonempty orthonormal basis of $H$, let $A: D(A) \subseteq$ $H \rightarrow H$ be a symmetric diagonal linear operator with $\inf \left(\sigma_{\mathrm{P}}(A)\right)>0$, and let $\left(H_{r},\langle\cdot, \cdot\rangle_{H_{r}},\|\cdot\|_{H_{r}}\right), r \in \mathbb{R}$, be a family of interpolation spaces associated to $A$. Then

(i) for all $v \in \bigcup_{s \in \mathbb{R}} H_{s}, r \in \mathbb{R}$ it holds that $v \in H_{r}$ if and only if

$$
\sup _{w \in \operatorname{span}_{H}(\mathbb{H}) \backslash\{0\}} \frac{\left|\langle w, v\rangle_{H}\right|}{\|w\|_{H_{-r}}}<\infty,
$$

(ii) for all $s \in \mathbb{R}, v \in H_{-s}, r \in[-s, \infty)$ it holds that $v \in H_{r}$ if and only if

$$
\sup _{w \in H_{s} \backslash\{0\}} \frac{\left|\langle w, v\rangle_{H}\right|}{\|w\|_{H_{-r}}}<\infty,
$$

and

(iii) for all $r \in \mathbb{R}, v \in H_{r}, s \in[-r, \infty)$ it holds that

$$
\|v\|_{H_{r}}=\sup _{w \in \operatorname{span}_{H}(\mathbb{H}) \backslash\{0\}} \frac{\left|\langle w, v\rangle_{H}\right|}{\|w\|_{H_{-r}}}
$$




$$
=\sup _{w \in H_{s} \backslash\{0\}} \frac{\left|\langle w, v\rangle_{H}\right|}{\|w\|_{H_{-r}}} .
$$

In the next result, Corollary 3.10, we specialize Corollary 3.7 above to the case of semilinear stochastic wave equations. Corollary 3.10 is an elementary consequence of Corollary 3.7.

Corollary 3.10 Consider the notation in Sect. 1.1, let T, $\vartheta \in(0, \infty), \gamma \in(1 / 4,1 / 2), \rho \in$ $[0,2 \gamma-1 / 2), \varrho \in[1 / 6, \infty)$, let $(\Omega, \mathcal{F}, \mathbb{P})$ be a probability space with a normal filtration $\left(\mathbb{F}_{t}\right)_{t \in[0, T]}$, let $\left(H,\langle\cdot, \cdot\rangle_{H},\|\cdot\|_{H}\right)=\left(L^{2}\left(\mu_{(0,1)} ; \mathbb{R}\right),\langle\cdot, \cdot\rangle_{L^{2}\left(\mu_{(0,1)} ; \mathbb{R}\right)},\|\cdot\|_{L^{2}\left(\mu_{(0,1)} ; \mathbb{R}\right)}\right)$, let $\left(W_{t}\right)_{t \in[0, T]}$ be an $\mathrm{id}_{H}$-cylindrical $\left(\Omega, \mathcal{F}, \mathbb{P},\left(\mathbb{F}_{t}\right)_{t \in[0, T]}\right)$-Wiener process, let $\left(e_{n}\right)_{n \in \mathbb{N}} \subseteq H$ satisfy for all $n \in \mathbb{N}$ and $\mu_{(0,1)}$-a.e. $x \in(0,1)$ that $e_{n}(x)=$ $\sqrt{2} \sin (n \pi x)$, let $A: D(A) \subseteq H \rightarrow H$ be the Laplacian with Dirichlet boundary conditions on $H$ multiplied by $\vartheta$, let $\left(H_{r},\langle\cdot, \cdot\rangle_{H_{r}},\|\cdot\|_{H_{r}}\right), r \in \mathbb{R}$, be a family of interpolation spaces associated to $-A$, let $\mathbf{P}_{N} \in L\left(H \times H_{-1 / 2}\right), N \in \mathbb{N} \cup\{\infty\}$, be the linear operators which satisfy for all $N \in \mathbb{N} \cup\{\infty\}, v, w \in H$ that $\mathbf{P}_{N}(v, w)=$ $\left(\sum_{n=1}^{N}\left\langle e_{n}, v\right\rangle_{H} e_{n}, \sum_{n=1}^{N}\left\langle e_{n}, w\right\rangle_{H} e_{n}\right)$, let $\mathbf{A}: D(\mathbf{A}) \subseteq H \times H_{-1 / 2} \rightarrow H \times H_{-1 / 2}$ be the linear operator which satisfies $D(\mathbf{A})=H_{1 / 2} \times H$ and $\forall(v, w) \in H_{1 / 2} \times H: \mathbf{A}(v, w)=$ $(w, A v)$, let $\xi \in L^{2}\left(\mathbb{P}_{\mathbb{F}_{0}} ; H_{1 / 2} \times H\right), \varphi \in C_{\mathrm{b}}^{2}\left(H \times H_{-1 / 2}, \mathbb{R}\right), f \in \operatorname{Lip}^{2}((0,1) \times \mathbb{R}, \mathbb{R})$, $B \in \operatorname{Lip}^{0}\left(H, L_{2}\left(H, H_{-1 / 2}\right)\right)$ satisfy $\forall v \in H_{\rho}, u \in H: B(v) u \in H_{\gamma-1 / 2}, \forall v \in$ $H_{\rho}:\left(H \ni u \mapsto B(v) u \in H_{\rho-1 / 2}\right) \in L_{2}\left(H, H_{\rho-1 / 2}\right),\left(H_{\rho} \ni v \mapsto(H \ni u \mapsto\right.$ $\left.\left.B(v) u \in H_{\rho-1 / 2}\right) \in L_{2}\left(H, H_{\rho-1 / 2}\right)\right) \in \operatorname{Lip}^{0}\left(H_{\rho}, L_{2}\left(H, H_{\rho-1 / 2}\right)\right), \forall v \in H_{\rho}:(H \ni$ $\left.u \mapsto B(v) u \in H_{\gamma-1 / 2}\right) \in L\left(H, H_{\gamma-1 / 2}\right),\left(H_{\rho} \ni v \mapsto\left(H \ni u \mapsto B(v) u \in H_{\gamma-1 / 2}\right) \in\right.$ $\left.L\left(H, H_{\gamma-1 / 2}\right)\right) \in \operatorname{Lip}^{0}\left(H_{\rho}, L\left(H, H_{\gamma-1 / 2}\right)\right),\left.B\right|_{H_{\varrho}} \in C_{\mathrm{b}}^{2}\left(H_{\varrho}, L_{2}\left(H, H_{-1 / 2}\right)\right)$, and $\sup _{x, v_{1}, v_{2} \in H_{\varrho}, \max \left\{\left\|v_{1}\right\|_{H},\left\|v_{2}\right\|_{H}\right\} \leq 1}\left\|B^{\prime \prime}(x)\left(v_{1}, v_{2}\right)\right\|_{L_{2}\left(H, H_{-1 / 2}\right)}<\infty$, and let $\mathbf{F}: H \times$ $H_{-1 / 2} \rightarrow H_{1 / 2} \times H$ and $\mathbf{B}: H \times H_{-1 / 2} \rightarrow L_{2}\left(H, H \times H_{-1 / 2}\right)$ be the functions which satisfy for all $v, u \in H, w \in H_{-1 / 2}$ and $\mu_{(0,1)}$-a.e. $x \in(0,1)$ that $(\mathbf{F}(v, w))(x)=(0, f(x, v(x)))$ and $\mathbf{B}(v, w) u=(0, B(v) u)$. Then

(i) it holds that $\mathbf{F} \in \operatorname{Lip}^{0}\left(H \times H_{-1 / 2}, H_{1 / 2} \times H\right),\left.\mathbf{F}\right|_{H_{Q} \times H_{Q-1 / 2}} \in \operatorname{Lip}^{2}\left(H_{Q} \times\right.$ $\left.H_{\varrho-1 / 2}, H_{1 / 2} \times H\right), \mathbf{B} \in \operatorname{Lip}^{0}\left(H \times H_{-1 / 2}, L_{2}\left(H, H \times H_{-1 / 2}\right)\right), \forall v \in H_{\rho} \times$ $H_{\rho-1 / 2}, u \in H: \mathbf{B}(v) u \in H_{\gamma} \times H_{\gamma-1 / 2}, \forall v \in H_{\rho} \times H_{\rho-1 / 2}:(H \ni u \mapsto$ $\left.\mathbf{B}(v) u \in H_{\rho} \times H_{\rho-1 / 2}\right) \in L_{2}\left(H, H_{\rho} \times H_{\rho-1 / 2}\right),\left(H_{\rho} \times H_{\rho-1 / 2} \ni v \mapsto\right.$ $\left.\left(H \ni u \mapsto \mathbf{B}(v) u \in H_{\rho} \times H_{\rho-1 / 2}\right) \in L_{2}\left(H, H_{\rho} \times H_{\rho-1 / 2}\right)\right) \in \operatorname{Lip}^{0}\left(H_{\rho} \times\right.$ $\left.H_{\rho-1 / 2}, L_{2}\left(H, H_{\rho} \times H_{\rho-1 / 2}\right)\right), \forall v \in H_{\rho} \times H_{\rho-1 / 2}:(H \ni u \mapsto \mathbf{B}(v) u \in$ $\left.H_{\gamma} \times H_{\gamma-1 / 2}\right) \in L\left(H, H_{\gamma} \times H_{\gamma-1 / 2}\right),\left(H_{\rho} \times H_{\rho-1 / 2} \ni v \mapsto(H \ni u \mapsto \mathbf{B}(v) u \in\right.$ $\left.\left.H_{\gamma} \times H_{\gamma-1 / 2}\right) \in L\left(H, H_{\gamma} \times H_{\gamma-1 / 2}\right)\right) \in \operatorname{Lip}^{0}\left(H_{\rho} \times H_{\rho-1 / 2}, L\left(H, H_{\gamma} \times H_{\gamma-1 / 2}\right)\right)$, $\left.\mathbf{B}\right|_{H_{Q} \times H_{\varrho-1 / 2}} \in C_{\mathrm{b}}^{2}\left(H_{\varrho} \times H_{\varrho-1 / 2}, L_{2}\left(H, H \times H_{-1 / 2}\right)\right)$, and

$$
\begin{aligned}
& \forall \delta \in(-\infty, 1 / 4): \\
& \sup _{\substack{x \in H_{\varrho} \times H_{\varrho-1 / 2}, v_{1}, v_{2} \in H_{\varrho} \times H_{\varrho-1 / 2} \backslash\{0\}}} \frac{\left\|\mathbf{F}^{\prime \prime}(x)\left(v_{1}, v_{2}\right)\right\|_{H_{\delta} \times H_{\delta-1 / 2}}+\left\|\mathbf{B}^{\prime \prime}(x)\left(v_{1}, v_{2}\right)\right\|_{L_{2}\left(H, H \times H_{-1 / 2}\right)}}{\left\|v_{1}\right\|_{H \times H_{-1 / 2}}\left\|v_{2}\right\|_{H \times H_{-1 / 2}}}<\infty,
\end{aligned}
$$

(ii) it holds that there exist up to modifications unique $\left(\mathbb{F}_{t}\right)_{t \in[0, T] \text {-predictable }}$ stochastic processes $X^{N}:[0, T] \times \Omega \rightarrow \mathbf{P}_{N}\left(H_{\rho} \times H_{\rho-1 / 2}\right), N \in \mathbb{N} \cup\{\infty\}$, such 
that for all $N \in \mathbb{N} \cup\{\infty\}, t \in[0, T]$ it holds that $\sup _{s \in[0, T]} \mathbb{E}\left[\left\|X_{s}^{N}\right\|_{H_{\rho} \times H_{\rho-1 / 2}}^{2}\right]<$ $\infty$ and $\mathbb{P}$-a.s. that

$$
X_{t}^{N}=\mathrm{e}^{t \mathbf{A}} \mathbf{P}_{N} \xi+\int_{0}^{t} \mathrm{e}^{(t-s) \mathbf{A}} \mathbf{P}_{N} \mathbf{F}\left(X_{s}^{N}\right) \mathrm{d} s+\int_{0}^{t} \mathrm{e}^{(t-s) \mathbf{A}} \mathbf{P}_{N} \mathbf{B}\left(X_{s}^{N}\right) \mathrm{d} W_{s},
$$

and

(iii) it holds for all $\varepsilon \in(4(1 / 2-\gamma), \infty)$ that there exists a real number $C \in[0, \infty)$ such that for all $N \in \mathbb{N}$ it holds that

$$
\left|\mathbb{E}\left[\varphi\left(X_{T}^{\infty}\right)\right]-\mathbb{E}\left[\varphi\left(X_{T}^{N}\right)\right]\right| \leq C \cdot N^{\varepsilon-1}
$$

Proof of Corollary 3.10 Throughout this proof let $f_{k, \ell}:(0,1) \times \mathbb{R} \rightarrow \mathbb{R}, k, \ell \in\{0,1,2\}$ with $k+\ell \leq 2$, be the functions which satisfy for all $k, \ell \in\{0,1,2\},(x, y) \in(0,1) \times \mathbb{R}$ with $k+\ell \leq 2$ that $f_{k, \ell}(x, y)=\left(\frac{\partial^{k+\ell}}{\partial x^{k} \partial y^{\ell}} f\right)(x, y)$ and let $F: H \rightarrow H$ be the function which satisfies for all $v \in H$ and $\mu_{(0,1)}$-a.e. $x \in(0,1)$ that $(F(v))(x)=f(x, v(x))$. Then note that for all $u, v \in H, w \in H_{-1 / 2}$ it holds that $\mathbf{F}(v, w)=(0, F(v))$ and

$$
\begin{aligned}
& \|F(u)-F(v)\|_{H}=\left(\int_{0}^{1}|f(x, u(x))-f(x, v(x))|^{2} \mathrm{~d} x\right)^{1 / 2} \\
& \leq|f|_{\operatorname{Lip}^{0}((0,1) \times \mathbb{R}, \mathbb{R})}\|u-v\|_{H} .
\end{aligned}
$$

This proves that $F \in \operatorname{Lip}^{0}(H, H)$. Hence, we obtain that $\mathbf{F} \in \operatorname{Lip}^{0}\left(H \times H_{-1 / 2}, H_{1 / 2} \times\right.$ $H)$. Next observe that the Sobolev embedding theorem ensures for all $\delta \in[1,6]$ that

$$
\sup _{w \in H_{\varrho} \backslash\{0\}} \frac{\|w\|_{L^{\delta}\left(\mu_{(0,1)} ; \mathbb{R}\right)}}{\|w\|_{H_{\varrho}}}<\infty
$$

Moreover, note that it holds for all $v, h \in H$ and $\mu_{(0,1)}$-a.e. $x \in(0,1)$ that

$$
\begin{aligned}
& \left|f(x, v(x)+h(x))-f(x, v(x))-f_{0,1}(x, v(x)) h(x)\right| \\
& \quad=\left|\int_{0}^{1}\left[f_{0,1}(x, v(x)+y h(x))-f_{0,1}(x, v(x))\right] h(x) \mathrm{d} y\right| \\
& \quad \leq|f|_{\operatorname{Lip}^{1}((0,1) \times \mathbb{R}, \mathbb{R})}|h(x)|^{2} .
\end{aligned}
$$


This, Hölder's inequality, and (3.53) imply for all $v \in H_{\varrho}, h \in H_{\varrho} \backslash\{0\}$ that

$$
\begin{aligned}
& \frac{1}{\|h\|_{H_{\varrho}}}\left(\int_{0}^{1}\left|f(x, v(x)+h(x))-f(x, v(x))-f_{0,1}(x, v(x)) h(x)\right|^{2} \mathrm{~d} x\right)^{1 / 2} \\
& \quad \leq|f|_{\operatorname{Lip}^{1}((0,1) \times \mathbb{R}, \mathbb{R})} \frac{\|h\|_{L^{4}\left(\mu_{(0,1)} ; \mathbb{R}\right)}^{2}}{\|h\|_{H_{\varrho}}} \\
& \quad \leq|f|_{\operatorname{Lip}^{1}((0,1) \times \mathbb{R}, \mathbb{R})}\left(\sup _{w \in H_{\varrho} \backslash\{0\}} \frac{\|w\|_{L^{4}\left(\mu_{(0,1)} ; \mathbb{R}\right)}}{\|w\|_{H_{\varrho}}}\right)^{2}\|h\|_{H_{\varrho}}<\infty
\end{aligned}
$$

In addition, observe that it holds for all $v, h \in H_{\varrho}$ that

$$
\begin{aligned}
& \left(\int_{0}^{1}\left|f_{0,1}(x, v(x)) h(x)\right|^{2} \mathrm{~d} x\right)^{1 / 2} \\
& \quad \leq|f|_{C_{\mathrm{b}}^{1}((0,1) \times \mathbb{R}, \mathbb{R})}\|h\|_{H} \leq|f|_{C_{\mathrm{b}}^{1}((0,1) \times \mathbb{R}, \mathbb{R})}\left(\sup _{w \in H_{\varrho} \backslash\{0\}} \frac{\|w\|_{H}}{\|w\|_{H_{\varrho}}}\right)\|h\|_{H_{\varrho}} \\
& \quad=|f|_{\operatorname{Lip}^{0}((0,1) \times \mathbb{R}, \mathbb{R})}\left(\sup _{w \in H_{\varrho} \backslash\{0\}} \frac{\|w\|_{H}}{\|w\|_{H_{\varrho}}}\right)\|h\|_{H_{\varrho}}<\infty .
\end{aligned}
$$

Inequalities (3.55)-(3.56) prove that $\left.F\right|_{H_{\varrho}}: H_{Q} \rightarrow H$ is Fréchet differentiable, that for all $v, h \in H_{\varrho}$ and $\mu_{(0,1)}$-a.e. $x \in(0,1)$ it holds that

$$
\left(F^{\prime}(v) h\right)(x)=f_{0,1}(x, v(x)) h(x),
$$

and that $\sup _{v \in H_{\varrho}}\left\|F^{\prime}(v)\right\|_{L\left(H_{\varrho}, H\right)} \leq|f|_{C_{\mathrm{b}}^{1}((0,1) \times \mathbb{R}, \mathbb{R})}<\infty$. Furthermore, Hölder's inequality and (3.53) show for all $u, v, h \in H_{\varrho}$ that

$$
\begin{aligned}
& \left\|\left(F^{\prime}(u)-F^{\prime}(v)\right) h\right\|_{H} \\
& \quad=\left(\int_{0}^{1}\left|\left[f_{0,1}(x, u(x))-f_{0,1}(x, v(x))\right] h(x)\right|^{2} \mathrm{~d} x\right)^{1 / 2} \\
& \quad \leq|f|_{\operatorname{Lip}^{1}((0,1) \times \mathbb{R}, \mathbb{R})}\|u-v\|_{L^{4}\left(\mu_{(0,1)} ; \mathbb{R}\right)}\|h\|_{L^{4}\left(\mu_{(0,1)} ; \mathbb{R}\right)} \\
& \quad \leq|f|_{\operatorname{Lip}^{1}((0,1) \times \mathbb{R}, \mathbb{R})}\left(\sup _{w \in H_{\varrho} \backslash\{0\}} \frac{\|w\|_{L^{4}\left(\mu_{(0,1)} ; \mathbb{R}\right)}}{\|w\|_{H_{\varrho}}}\right)^{2}\|u-v\|_{H_{\varrho}}\|h\|_{H_{\varrho}}<\infty .
\end{aligned}
$$

This ensures that $\left.F\right|_{H_{\varrho}} \in \operatorname{Lip}^{1}\left(H_{\varrho}, H\right)$. Similarly, observe that for all $v, h, g \in H$ and $\mu_{(0,1)}$-a.e. $x \in(0,1)$ it holds that

$$
\begin{aligned}
& \left|f_{0,1}(x, v(x)+g(x)) h(x)-f_{0,1}(x, v(x)) h(x)-f_{0,2}(x, v(x)) h(x) g(x)\right| \\
& \quad=\left|\int_{0}^{1}\left[f_{0,2}(x, v(x)+y g(x))-f_{0,2}(x, v(x))\right] h(x) g(x) \mathrm{d} y\right| \\
& \quad \leq|f|_{\operatorname{Lip}^{2}((0,1) \times \mathbb{R}, \mathbb{R})}|h(x)||g(x)|^{2} .
\end{aligned}
$$


This, Hölder's inequality, and (3.53) establish for all $v, h \in H_{\varrho}, g \in H_{\varrho} \backslash\{0\}$ that

$$
\begin{aligned}
& \frac{1}{\|g\|_{H_{\varrho}}}\left(\int_{0}^{1} \mid f_{0,1}(x, v(x)+g(x)) h(x)\right. \\
& \left.-f_{0,1}(x, v(x)) h(x)-\left.f_{0,2}(x, v(x)) h(x) g(x)\right|^{2} \mathrm{~d} x\right)^{1 / 2} \\
& \leq \frac{|f|_{\operatorname{Lip}^{2}((0,1) \times \mathbb{R}, \mathbb{R})}}{\|g\|_{H_{\varrho}}}\left(\int_{0}^{1}|h(x)|^{2}|g(x)|^{4} \mathrm{~d} x\right)^{1 / 2} \\
& \leq|f|_{\operatorname{Lip}^{2}((0,1) \times \mathbb{R}, \mathbb{R})} \frac{\|h\|_{L^{6}\left(\mu_{(0,1)} ; \mathbb{R}\right)}\|g\|_{L^{6}\left(\mu_{(0,1)} ; \mathbb{R}\right)}^{2}}{\|g\|_{H_{\varrho}}} \\
& \leq|f|_{\operatorname{Lip}^{2}((0,1) \times \mathbb{R}, \mathbb{R})}\left(\sup _{w \in H_{\varrho} \backslash\{0\}} \frac{\|w\|_{L^{6}\left(\mu_{(0,1)} ; \mathbb{R}\right)}}{\|w\|_{H_{\varrho}}}\right)^{3}\|h\|_{H_{\varrho}}\|g\|_{H_{\varrho}}<\infty .
\end{aligned}
$$

Furthermore, Hölder's inequality and (3.53) also prove for all $v, h, g \in H_{\varrho}$ that

$$
\begin{aligned}
& \left(\int_{0}^{1}\left|f_{0,2}(x, v(x)) h(x) g(x)\right|^{2} \mathrm{~d} x\right)^{1 / 2} \leq|f|_{C_{\mathrm{b}}^{2}((0,1) \times \mathbb{R}, \mathbb{R})}\|h\|_{L^{4}\left(\mu_{(0,1)} ; \mathbb{R}\right)}\|g\|_{L^{4}\left(\mu_{(0,1)} ; \mathbb{R}\right)} \\
& \leq|f|_{C_{\mathrm{b}}^{2}((0,1) \times \mathbb{R}, \mathbb{R})}\left(\sup _{w \in H_{\varrho} \backslash\{0\}} \frac{\|w\|_{L^{4}\left(\mu_{(0,1)} ; \mathbb{R}\right)}}{\|w\|_{H_{\varrho}}}\right)^{2}\|h\|_{H_{\varrho}}\|g\|_{H_{\varrho}} \\
& =|f|_{\operatorname{Lip}^{1}((0,1) \times \mathbb{R}, \mathbb{R})}\left(\sup _{w \in H_{\varrho} \backslash\{0\}} \frac{\|w\|_{L^{4}\left(\mu_{(0,1)} ; \mathbb{R}\right)}}{\|w\|_{H_{\varrho}}}\right)^{2}\|h\|_{H_{\varrho}}\|g\|_{H_{\varrho}}<\infty
\end{aligned}
$$

Combining (3.60)-(3.61) ensures that $\left.F\right|_{H_{\varrho}}: H_{\varrho} \rightarrow H$ is twice Fréchet differentiable, that for all $v, h, g \in H_{\varrho}$ and $\mu_{(0,1)}$-a.e. $x \in(0,1)$ it holds that

$$
\left(F^{\prime \prime}(v)(h, g)\right)(x)=f_{0,2}(x, v(x)) h(x) g(x),
$$

and that

$$
\sup _{v \in H_{\varrho}}\left\|F^{\prime \prime}(v)\right\|_{L^{(2)}\left(H_{\varrho}, H\right)} \leq|f|_{C_{\mathrm{b}}^{2}((0,1) \times \mathbb{R}, \mathbb{R})}\left(\sup _{w \in H_{\varrho} \backslash\{0\}} \frac{\|w\|_{L^{4}\left(\mu_{(0,1)} ; \mathbb{R}\right)}}{\|w\|_{H_{\varrho}}}\right)^{2}<\infty .
$$

In addition, Hölder's inequality and (3.53) establish for all $u, v, h, g \in H_{\varrho}$ that

$$
\begin{aligned}
& \left\|\left(F^{\prime \prime}(u)-F^{\prime \prime}(v)\right)(h, g)\right\|_{H}=\left(\int_{0}^{1}\left|\left[f_{0,2}(x, u(x))-f_{0,2}(x, v(x))\right] h(x) g(x)\right|^{2} \mathrm{~d} x\right)^{1 / 2} \\
& \leq|f|_{\operatorname{Lip}^{2}((0,1) \times \mathbb{R}, \mathbb{R})}\|u-v\|_{L^{6}\left(\mu_{(0,1)} ; \mathbb{R}\right)}\|h\|_{L^{6}\left(\mu_{(0,1)} ; \mathbb{R}\right)}\|g\|_{L^{6}\left(\mu_{(0,1)} ; \mathbb{R}\right)} \\
& \leq|f|_{\operatorname{Lip}^{2}((0,1) \times \mathbb{R}, \mathbb{R})}\left(\sup _{w \in H_{\varrho} \backslash\{0\}} \frac{\|w\|_{L^{6}\left(\mu_{(0,1)} ; \mathbb{R}\right)}}{\|w\|_{H_{Q}}}\right)^{3}\|u-v\|_{H_{\varrho}}\|h\|_{H_{\varrho}}\|g\|_{H_{Q}}<\infty .
\end{aligned}
$$

This shows that $\left.F\right|_{H_{\varrho}} \in \operatorname{Lip}^{2}\left(H_{\varrho}, H\right)$. This proves that $\left.\mathbf{F}\right|_{H_{\varrho} \times H_{\varrho-1 / 2}} \in \operatorname{Lip}^{2}\left(H_{\varrho} \times\right.$ $\left.H_{\varrho-1 / 2}, H_{1 / 2} \times H\right)$. Next, note that the assumptions that $B \in \operatorname{Lip}^{0}\left(H, L_{2}\left(H, H_{-1 / 2}\right)\right)$, 


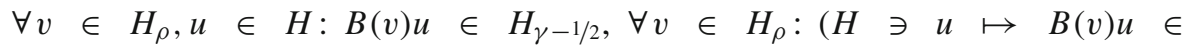
$\left.H_{\rho-1 / 2}\right) \in L_{2}\left(H, H_{\rho-1 / 2}\right),\left(H_{\rho} \ni v \mapsto\left(H \ni u \mapsto B(v) u \in H_{\rho-1 / 2}\right) \in\right.$ $\left.L_{2}\left(H, H_{\rho-1 / 2}\right)\right) \in \operatorname{Lip}^{0}\left(H_{\rho}, L_{2}\left(H, H_{\rho-1 / 2}\right)\right), \forall v \in H_{\rho}:(H \ni u \mapsto B(v) u \in$ $\left.H_{\gamma-1 / 2}\right) \in L\left(H, H_{\gamma-1 / 2}\right),\left(H_{\rho} \ni v \mapsto\left(H \ni u \mapsto B(v) u \in H_{\gamma-1 / 2}\right) \in\right.$ $\left.L\left(H, H_{\gamma-1 / 2}\right)\right) \in \operatorname{Lip}^{0}\left(H_{\rho}, L\left(H, H_{\gamma-1 / 2}\right)\right)$, and $\left.B\right|_{H_{\varrho}} \in C_{\mathrm{b}}^{2}\left(H_{\varrho}, L_{2}\left(H, H_{-1 / 2}\right)\right)$ ensure that $\mathbf{B} \in \operatorname{Lip}^{0}\left(H \times H_{-1 / 2}, L_{2}\left(H, H \times H_{-1 / 2}\right)\right), \forall v \in H_{\rho} \times H_{\rho-1 / 2}, u \in$ $H: \mathbf{B}(v) u \in H_{\gamma} \times H_{\gamma-1 / 2}, \forall v \in H_{\rho} \times H_{\rho-1 / 2}:\left(H \ni u \mapsto \mathbf{B}(v) u \in H_{\rho} \times\right.$ $\left.H_{\rho-1 / 2}\right) \in L_{2}\left(H, H_{\rho} \times H_{\rho-1 / 2}\right),\left(H_{\rho} \times H_{\rho-1 / 2} \ni v \mapsto(H \ni u \mapsto \mathbf{B}(v) u \in\right.$ $\left.\left.H_{\rho} \times H_{\rho-1 / 2}\right) \in L_{2}\left(H, H_{\rho} \times H_{\rho-1 / 2}\right)\right) \in \operatorname{Lip}^{0}\left(H_{\rho} \times H_{\rho-1 / 2}, L_{2}\left(H, H_{\rho} \times H_{\rho-1 / 2}\right)\right)$, $\forall v \in H_{\rho} \times H_{\rho-1 / 2}:\left(H \ni u \mapsto \mathbf{B}(v) u \in H_{\gamma} \times H_{\gamma-1 / 2}\right) \in L\left(H, H_{\gamma} \times H_{\gamma-1 / 2}\right)$, $\left(H_{\rho} \times H_{\rho-1 / 2} \ni v \mapsto\left(H \ni u \mapsto \mathbf{B}(v) u \in H_{\gamma} \times H_{\gamma-1 / 2}\right) \in L\left(H, H_{\gamma} \times\right.\right.$ $\left.\left.H_{\gamma-1 / 2}\right)\right) \in \operatorname{Lip}^{0}\left(H_{\rho} \times H_{\rho-1 / 2}, L\left(H, H_{\gamma} \times H_{\gamma-1 / 2}\right)\right)$, and $\left.\mathbf{B}\right|_{H_{\varrho} \times H_{O-1 / 2}} \in C_{\mathrm{b}}^{2}\left(H_{\varrho} \times\right.$ $\left.H_{\varrho-1 / 2}, L_{2}\left(H, H \times H_{-1 / 2}\right)\right)$. In addition, Lemma 3.9 proves for all $\delta \in(-\infty, 1 / 4)$, $v, h, g \in H_{\varrho}$ that

$$
\begin{aligned}
& \left\|F^{\prime \prime}(v)(h, g)\right\|_{H_{\delta-1 / 2}}=\sup _{w \in H_{1 / 2-\delta} \backslash\{0\}} \frac{\left\langle w, F^{\prime \prime}(v)(h, g)\right\rangle_{H}}{\|w\|_{H_{1 / 2-\delta}}} \\
& \leq|f|_{C_{\mathrm{b}}^{2}((0,1) \times \mathbb{R}, \mathbb{R})}\left(\sup _{w \in H_{1 / 2-\delta} \backslash\{0\}} \frac{\|w\|_{L^{\infty}\left(\mu_{(0,1)} ; \mathbb{R}\right)}}{\|w\|_{H_{1 / 2-\delta}}}\right)\|h\|_{H}\|g\|_{H}<\infty .
\end{aligned}
$$

This and the assumption that

$$
\sup _{x, v_{1}, v_{2} \in H_{\varrho}, \max \left\{\left\|v_{1}\right\|_{H},\left\|v_{2}\right\|_{H}\right\} \leq 1}\left\|B^{\prime \prime}(x)\left(v_{1}, v_{2}\right)\right\|_{L_{2}\left(H, H_{-1 / 2}\right)}<\infty
$$

show (3.49). The proof of (i) is thus complete. Furthermore, observe that (ii) follows directly from (i) and Remark 3.1. It thus remains to prove (iii). For this let $\varepsilon \in$ $(4(1 / 2-\gamma), 1-2 \rho], \beta \in(1 / 2,2 \gamma]$ and $\lambda_{n} \in \mathbb{R}, n \in \mathbb{N}$, be real numbers which satisfy for all $n \in \mathbb{N}$ that $\beta=1 / 2+(\varepsilon-4(1 / 2-\gamma)) / 2$ and $\lambda_{n}=-\vartheta \pi^{2} n^{2}$ and let $\boldsymbol{\Lambda}: D(\boldsymbol{\Lambda}) \subseteq$ $H \times H_{-1 / 2} \rightarrow H \times H_{-1 / 2}$ be the linear operator which satisfies for all $(v, w) \in H_{1 / 2} \times H$ that $D(\boldsymbol{\Lambda})=H_{1 / 2} \times H$ and

$$
\boldsymbol{\Lambda}(v, w)=\left(\begin{array}{c}
\sum_{n=1}^{\infty}\left|\lambda_{n}\right|^{1 / 2}\left\langle e_{n}, v\right\rangle_{H} e_{n} \\
\sum_{n=1}^{\infty}\left|\lambda_{n}\right|^{1 / 2}\left\langle e_{n}, w\right\rangle_{H} e_{n}
\end{array}\right) .
$$

Then note that for all $v \in H_{1}$ it holds that $A v=\sum_{n=1}^{\infty} \lambda_{n}\left\langle e_{n}, v\right\rangle_{H} e_{n}$ and $\left\|\boldsymbol{\Lambda}^{-\beta}\right\|_{L_{2}\left(H \times H_{-1 / 2}\right)}<\infty$. Furthermore, observe that (i) and the fact that $2 \gamma-\beta=$ $(1-\varepsilon) / 2 \leq 1 / 2$ imply that $\left(H \times H_{-1 / 2} \ni v \mapsto \mathbf{F}(v) \in H_{2 \gamma-\beta} \times H_{2 \gamma-\beta-1 / 2}\right) \in$ $\operatorname{Lip}^{0}\left(H \times H_{-1 / 2}, H_{2 \gamma-\beta} \times H_{2 \gamma-\beta-1 / 2}\right)$. This, the fact that $2 \rho \leq 1-\varepsilon=2(2 \gamma-\beta)$, and again (i) enable us to apply Corollary 3.7 to obtain that there exists a real number $C \in[0, \infty)$ such that for all $N \in \mathbb{N}$ it holds that 


$$
\left|\mathbb{E}\left[\varphi\left(X_{T}^{\infty}\right)\right]-\mathbb{E}\left[\varphi\left(X_{T}^{N}\right)\right]\right| \leq C\left|\lambda_{N+1}\right|^{\beta-2 \gamma} \leq C \vartheta^{(\varepsilon-1) / 2} \cdot N^{\varepsilon-1}
$$

The proof of Corollary 3.10 is thus complete.

In the proof of Corollary 3.12 below we employ the following elementary and well-known result, Lemma 3.11.

Lemma 3.11 Let $\mathbb{K} \in\{\mathbb{R}, \mathbb{C}\}$, let $\left(H,\langle\cdot, \cdot\rangle_{H},\|\cdot\|_{H}\right)$ be a $\mathbb{K}$-Hilbert space, let $\mathbb{H} \subseteq H$ be a nonempty orthonormal basis of $H$, let $A: D(A) \subseteq H \rightarrow H$ be a symmetric diagonal linear operator with $\inf \left(\sigma_{\mathrm{P}}(A)\right)>0$, let $\left(H_{r},\langle\cdot, \cdot\rangle_{H_{r}},\|\cdot\|_{H_{r}}\right), r \in \mathbb{R}$, be a family of interpolation spaces associated to $A$, and let $q, s \in \mathbb{R}, p \in[q, \infty)$, $r \in[s, \infty)$. Then

(i) for all $B \in L\left(H_{q}, H_{s}\right)$ it holds that $\left(B\left(H_{q}\right) \subseteq H_{r}\right.$ and $\left(H_{q} \ni v \mapsto B v \in H_{r}\right) \in$ $\left.L\left(H_{q}, H_{r}\right)\right)$ if and only if

$$
\left(B\left(\operatorname{span}_{H}(\mathbb{H})\right) \subseteq H_{r} \quad \text { and } \sup _{w \in \operatorname{span}_{H}(\mathbb{H}) \backslash\{0\}} \frac{\|B w\|_{H_{r}}}{\|w\|_{H_{q}}}<\infty\right),
$$

(ii) for all $B \in L\left(H_{q}, H_{s}\right)$ it holds that $\left(B\left(H_{q}\right) \subseteq H_{r}\right.$ and $\left(H_{q} \ni v \mapsto B v \in H_{r}\right) \in$ $\left.L\left(H_{q}, H_{r}\right)\right)$ if and only if

$$
\left(B\left(H_{p}\right) \subseteq H_{r} \quad \text { and } \sup _{w \in H_{p} \backslash\{0\}} \frac{\|B w\|_{H_{r}}}{\|w\|_{H_{q}}}<\infty\right)
$$

and

(iii) for all $B \in L\left(H_{q}, H_{r}\right)$ it holds that

$$
\|B\|_{L\left(H_{q}, H_{r}\right)}=\sup _{w \in \operatorname{span}_{H}(\mathbb{H}) \backslash\{0\}} \frac{\|B w\|_{H_{r}}}{\|w\|_{H_{q}}}=\sup _{w \in H_{p} \backslash\{0\}} \frac{\|B w\|_{H_{r}}}{\|w\|_{H_{q}}} .
$$

Corollary 3.12 Consider the notation in Sect. 1.1, let $T, \vartheta \in(0, \infty), \alpha, \beta \in$ $\mathbb{R}$, let $(\Omega, \mathcal{F}, \mathbb{P})$ be a probability space with a normal filtration $\left(\mathbb{F}_{t}\right)_{t \in[0, T]}$, let $\left(H,\langle\cdot, \cdot\rangle_{H},\|\cdot\|_{H}\right)=\left(L^{2}\left(\mu_{(0,1)} ; \mathbb{R}\right),\langle\cdot, \cdot\rangle_{L^{2}\left(\mu_{(0,1)} ; \mathbb{R}\right)},\|\cdot\|_{L^{2}\left(\mu_{(0,1)} ; \mathbb{R}\right)}\right)$, let $\left(W_{t}\right)_{t \in[0, T]}$ be an $\operatorname{id}_{H}$-cylindrical $\left(\Omega, \mathcal{F}, \mathbb{P},\left(\mathbb{F}_{t}\right)_{t \in[0, T]}\right)$-Wiener process, let $\left(e_{n}\right)_{n \in \mathbb{N}} \subseteq H$ satisfy for all $n \in \mathbb{N}$ and $\mu_{(0,1)}$-a.e. $x \in(0,1)$ that $e_{n}(x)=\sqrt{2} \sin (n \pi x)$, let $A: D(A) \subseteq$ $H \rightarrow H$ be the Laplacian with Dirichlet boundary conditions on $H$ multiplied by $\vartheta$, let $\left(H_{r},\langle\cdot, \cdot\rangle_{H_{r}},\|\cdot\|_{H_{r}}\right), r \in \mathbb{R}$, be a family of interpolation spaces associated to $-A$, let $\mathbf{P}_{N} \in L\left(H \times H_{-1 / 2}\right), N \in \mathbb{N} \cup\{\infty\}$, be the linear operators which satisfy for all $N \in \mathbb{N} \cup\{\infty\}, v, w \in H$ that $\mathbf{P}_{N}(v, w)=\left(\sum_{n=1}^{N}\left\langle e_{n}, v\right\rangle_{H} e_{n}, \sum_{n=1}^{N}\left\langle e_{n}, w\right\rangle_{H} e_{n}\right)$, let $\mathbf{A}: D(\mathbf{A}) \subseteq H \times H_{-1 / 2} \rightarrow H \times H_{-1 / 2}$ be the linear operator which satisfies $D(\mathbf{A})=H_{1 / 2} \times H$ and $\forall(v, w) \in H_{1 / 2} \times H: \mathbf{A}(v, w)=(w, A v)$, let $\xi \in L^{2}\left(\left.\mathbb{P}\right|_{F_{0}} ; H_{1 / 2} \times H\right), \varphi \in C_{\mathrm{b}}^{2}\left(H \times H_{-1 / 2}, \mathbb{R}\right), f \in \operatorname{Lip}^{2}((0,1) \times \mathbb{R}, \mathbb{R})$, and let $\mathbf{F}: H \times H_{-1 / 2} \rightarrow H_{1 / 2} \times H$ and $\mathbf{B}: H \times H_{-1 / 2} \rightarrow L_{2}\left(H, H \times H_{-1 / 2}\right)$ be the functions which satisfy for all $(v, w) \in H \times H_{-1 / 2}, u \in H_{1}$ and $\mu_{(0,1)}$-a.e. $x \in(0,1)$ that $(\mathbf{F}(v, w))(x)=(0, f(x, v(x)))$ and $(\mathbf{B}(v, w) u)(x)=(0,(\alpha+\beta v(x)) u(x))$. Then 
(i) it holds that there exist up to modifications unique $\left(\mathbb{F}_{t}\right)_{t \in[0, T] \text {-predictable }}$ stochastic processes $X^{N}:[0, T] \times \Omega \rightarrow\left(\bigcap_{\rho \in[0,1 / 4)} \mathbf{P}_{N}\left(H_{\rho} \times H_{\rho-1 / 2}\right)\right), N \in$ $\mathbb{N} \cup\{\infty\}$, such that for all $\rho \in[0,1 / 4), N \in \mathbb{N} \cup\{\infty\}, t \in[0, T]$ it holds that $\sup _{s \in[0, T]} \mathbb{E}\left[\left\|X_{s}^{N}\right\|_{H_{\rho} \times H_{\rho-1 / 2}}^{2}\right]<\infty$ and $\mathbb{P}$-a.s. that

$$
X_{t}^{N}=\mathrm{e}^{t \mathbf{A}} \mathbf{P}_{N} \xi+\int_{0}^{t} \mathrm{e}^{(t-s) \mathbf{A}} \mathbf{P}_{N} \mathbf{F}\left(X_{s}^{N}\right) \mathrm{d} s+\int_{0}^{t} \mathrm{e}^{(t-s) \mathbf{A}} \mathbf{P}_{N} \mathbf{B}\left(X_{s}^{N}\right) \mathrm{d} W_{s}
$$

and

(ii) it holds for all $\varepsilon \in(0, \infty)$ that there exists a real number $C \in[0, \infty)$ such that for all $N \in \mathbb{N}$ it holds that

$$
\left|\mathbb{E}\left[\varphi\left(X_{T}^{\infty}\right)\right]-\mathbb{E}\left[\varphi\left(X_{T}^{N}\right)\right]\right| \leq C \cdot N^{\varepsilon-1}
$$

Proof of Corollary 3.12 Throughout this proof let $B: H \rightarrow L_{2}\left(H, H_{-1 / 2}\right)$ be the function which satisfies for all $v \in H, u \in H_{1}$ and $\mu_{(0,1)}$-a.e. $x \in(0,1)$ that $(B(v) u)(x)=(\alpha+\beta v(x)) u(x)$. Note that it holds for all $\rho \in[0,1 / 4), v, u \in H$ that $B(v) u \in H_{\rho-1 / 2},\left(H \ni y \mapsto B(v) y \in H_{\rho-1 / 2}\right) \in L_{2}\left(H, H_{\rho-1 / 2}\right)$, and $(H \ni$ $\left.x \mapsto\left(H \ni y \mapsto B(x) y \in H_{\rho-1 / 2}\right) \in L_{2}\left(H, H_{\rho-1 / 2}\right)\right) \in \operatorname{Lip}^{0}\left(H, L_{2}\left(H, H_{\rho-1 / 2}\right)\right)$. Remark 3.1 and (i) in Corollary 3.10 thus prove (i). Next observe that the Sobolev embedding theorem proves for all $\rho \in(0,1 / 4)$ that

$$
\left[\sup _{w \in H_{1} \backslash\{0\}} \frac{\|w\|_{L^{1 /(2 \rho)}\left(\mu_{(0,1)} ; \mathbb{R}\right)}}{\|w\|_{H_{1 / 4-\rho}}}\right]+\left[\sup _{w \in H_{\rho} \backslash\{0\}} \frac{\|w\|_{L^{2 /(1-4 \rho)}\left(\mu_{(0,1)} ; \mathbb{R}\right)}}{\|w\|_{H_{\rho}}}\right]<\infty
$$

This and Hölder's inequality ensure for all $\rho \in(0,1 / 4), v \in H_{\rho}, u \in H_{1}$ that

$$
\begin{aligned}
& \sup _{w \in H_{1} \backslash\{0\}} \frac{\left|\langle w, B(v) u\rangle_{H}\right|}{\|w\|_{H_{1 / 4-\rho}}} \\
& \leq\left[\sup _{w \in H_{1} \backslash\{0\}} \frac{\|w\|_{L^{1 /(2 \rho)}\left(\mu_{(0,1)} ; \mathbb{R}\right)}}{\|w\|_{H_{1 / 4-\rho}}}\right]\|\alpha+\beta v\|_{L^{2 /(1-4 \rho)}\left(\mu_{(0,1)} ; \mathbb{R}\right)}\|u\|_{L^{2}\left(\mu_{(0,1)} ; \mathbb{R}\right)} \\
& \leq\left[\sup _{w \in H_{1} \backslash\{0\}} \frac{\|w\|_{L^{1 /(2 \rho)}\left(\mu_{(0,1)} ; \mathbb{R}\right)}}{\|w\|_{H_{1 / 4-\rho}}}\right]\left[\sup _{w \in H_{\rho} \backslash\{0\}} \frac{\|w\|_{L^{2 /(1-4 \rho)}\left(\mu_{(0,1)} ; \mathbb{R}\right)}}{\|w\|_{H_{\rho}}}\right]\|\alpha+\beta v\|_{H_{\rho}}\|u\|_{H}<\infty .
\end{aligned}
$$

Lemma 3.9 hence shows for all $\rho \in(0,1 / 4), v \in H_{\rho}, u \in H_{1}$ that $B(v) u \in H_{\rho-1 / 4}$. In addition, (3.75) and Lemmas 3.9 and 3.11 prove for all $\rho \in(0,1 / 4), v \in H_{\rho}, u \in H$ that $B(v) u \in H_{\rho-1 / 4}$ and $\left(H \ni y \mapsto B(v) y \in H_{\rho-1 / 4}\right) \in L\left(H, H_{\rho-1 / 4}\right)$. Furthermore, Lemma 3.9 and Hölder's inequality show for all $\rho \in(0,1 / 4), v_{1}, v_{2} \in H_{\rho}, u \in H_{1}$ that 


$$
\begin{aligned}
& \left\|\left(B\left(v_{1}\right)-B\left(v_{2}\right)\right) u\right\|_{H_{\rho-1 / 4}}=\sup _{w \in H_{1} \backslash\{0\}} \frac{\left|\left\langle w,\left(B\left(v_{1}\right)-B\left(v_{2}\right)\right) u\right\rangle_{H}\right|}{\|w\|_{H_{1 / 4-\rho}}} \\
& \leq\left[\sup _{w \in H_{1} \backslash\{0\}} \frac{\|w\|_{L^{1 /(2 \rho)}\left(\mu_{(0,1)} ; \mathbb{R}\right)}}{\|w\|_{H_{1 / 4-\rho}}}\right]\left[\sup _{w \in H_{\rho} \backslash\{0\}} \frac{\|w\|_{L^{2 /(1-4 \rho)}\left(\mu_{(0,1)} ; \mathbb{R}\right)}}{\|w\|_{H_{\rho}}}\right] \\
& \quad \cdot|\beta|\left\|v_{1}-v_{2}\right\|_{H_{\rho}}\|u\|_{H}<\infty .
\end{aligned}
$$

This and Lemma 3.11 establish for all $\varepsilon \in(0,1], \gamma \in(1 / 2-\varepsilon / 4,1 / 2), \rho \in$ $[\gamma-1 / 4, \min \{2 \gamma-1 / 2,1 / 4\})$ that $\left(H_{\rho} \ni v \mapsto\left(H \ni u \mapsto B(v) u \in H_{\gamma-1 / 2}\right) \in\right.$ $\left.L\left(H, H_{\gamma-1 / 2}\right)\right) \in \operatorname{Lip}^{0}\left(H_{\rho}, L\left(H, H_{\gamma-1 / 2}\right)\right)$. Corollary 3.10 thus completes the proof of Corollary 3.12.

Acknowledgements This project has been partially supported through the ETH Research Grant ETH-47 15-2 "Mild stochastic calculus and numerical approximations for nonlinear stochastic evolution equations with Lévy noise" and through the SNSF-Research project 200021_156603 "Numerical approximations of nonlinear stochastic ordinary and partial differential equations".

Funding Open Access funding provided by ETH Zurich.

Open Access This article is licensed under a Creative Commons Attribution 4.0 International License, which permits use, sharing, adaptation, distribution and reproduction in any medium or format, as long as you give appropriate credit to the original author(s) and the source, provide a link to the Creative Commons licence, and indicate if changes were made. The images or other third party material in this article are included in the article's Creative Commons licence, unless indicated otherwise in a credit line to the material. If material is not included in the article's Creative Commons licence and your intended use is not permitted by statutory regulation or exceeds the permitted use, you will need to obtain permission directly from the copyright holder. To view a copy of this licence, visit http://creativecommons.org/licenses/by/4.0/.

\section{References}

1. Andersson, A., Kovács, M., Larsson, S: Weak error analysis for semilinear stochastic Volterra equations with additive noise. J. Math. Anal. Appl. 437(2), 1283-1304 (2016). https://doi.org/10.1016/j.jmaa. 2015.09.016

2. Andersson, A., Larsson, S.: Weak convergence for a spatial approximation of the nonlinear stochastic heat equation. Math. Comp. 85(299), 1335-1358 (2016). https://doi.org/10.1090/mcom/3016

3. Anton, R., Cohen, D., Larsson, S., Wang, X.: Full discretization of semilinear stochastic wave equations driven by multiplicative noise. SIAM J. Numer. Anal. 54(2), 1093-1119 (2016). https://doi.org/10. 1137/15M101049X

4. Bréhier, C-E.: Strong and weak orders in averaging for SPDEs. Stoch. Process. Appl. 122(7), 25532593 (2012). https://doi.org/10.1016/j.spa.2012.04.007

5. Bréhier, C.-E.: Approximation of the invariant measure with an Euler scheme for stochastic PDEs driven by space-time white noise. Potential Anal. 40(1), 1-40 (2014). https://doi.org/10.1007/s11118013-9338-9

6. Bréhier, C.-E., Kopec, M.: Approximation of the invariant law of SPDEs: error analysis using a Poisson equation for a full-discretization scheme. IMA J. Numer. Anal. 37(3), 1375-1410 (2017). https://doi. org/10.1093/imanum/drw030

7. Cohen, D., Larsson, S., Sigg, M.: A trigonometric method for the linear stochastic wave equation. SIAM J. Numer. Anal. 51(1), 204-222 (2013). https://doi.org/10.1137/12087030X

8. Cohen, D., Quer-Sardanyons, L.: A fully discrete approximation of the one-dimensional stochastic wave equation. IMA J. Numer. Anal. 36(1), 400-420 (2016). https://doi.org/10.1093/imanum/drv006

9. Conus, D., Jentzen, A., Kurniawan, R.: Weak convergence rates of spectral Galerkin approximations for SPDEs with nonlinear diffusion coefficients. Ann. Appl. Probab. 29(2), 653-716 (2019). https:// doi.org/10.1214/17-AAP1352 
10. Conus, D., Joseph, M., Khoshnevisan, D., Shiu, S.-Y.: Intermittency and Chaos for a Nonlinear Stochastic Wave Equation in Dimension 1. In: Malliavin Calculus and Stochastic Analysis. Vol. 34. Springer Proc. Math. Stat. Springer, New York, 2013, 251-279. ISBN: 978-1-4614-5906-4. https://doi.org/10. 1007/978-1-4614-5906-4_11

11. Cox, S., Jentzen, A., Lindner, F.: Weak convergence rates for temporal numerical approximations of stochastic wave equations with multiplicative noise. ArXiv e-prints (2019), 51 pages. arXiv:1901.05535 [math.PR]

12. Da Prato, G., Jentzen, A., Röckner, M.: A mild Itô formula for SPDEs. Trans. Am. Math. Soc. 372(6), 3755-3807 (2019). https://doi.org/10.1090/tran/7165

13. Da Prato, G., Zabczyk, J.: Stochastic Equations in Infinite Dimensions. Vol. 44. Encyclopedia of Mathematics and its Applications. Cambridge Univ. Press, Cambridge, 1992, xviii+454. ISBN: 0521-38529-6. https://doi.org/10.1017/CBO9780511666223

14. Dalang, R.C.: The stochastic wave equation. In: A Minicourse on Stochastic Partial Differential Equations. Ed. by Khoshnevisan, D., Rassoul-Agha, F.: Vol. 1962. Lecture Notes in Mathematics. Springer Berlin Heidelberg, 2009, 39-71. ISBN: 978-3-540- 85993-2. https://doi.org/10.1007/978-3540-85994-9_2

15. Davie, A.M., Gaines, J.G.: Convergence of numerical schemes for the solution of parabolic stochastic partial differential equations. Math. Comp. 70(233), 121-134 (2001). https://doi.org/10.1090/S00255718-00-01224-2

16. de Bouard, A., Debussche, A.: Weak and strong order of convergence of a semidiscrete scheme for the stochastic nonlinear Schrödinger equation. Appl. Math. Optim. 54(3), 369-399 (2006). https://doi. org/10.1007/s00245-006-0875-0

17. Debussche, A.: Weak approximation of stochastic partial differential equations: the nonlinear case. Math. Comp. 80(273), 89-117 (2011). https://doi.org/10.1090/S0025-5718-2010-02395-6

18. Debussche, A., Printems, J.: Weak order for the discretization of the stochastic heat equation. Math. Comp. 78(266), 845-863 (2009). https://doi.org/10.1090/S0025-5718-08-02184-4

19. Geissert, M., Kovács, M., Larsson, S.: Rate of weak convergence of the finite element method for the stochastic heat equation with additive noise. BIT 49(2), 343-356 (2009). https://doi.org/10.1007/ s10543-009-0227-y

20. Harms, P., Müller, M.S.: Weak convergence rates for stochastic evolution equations and applications to nonlinear stochastic wave, HJMM, stochastic Schrödinger and linearized stochastic Korteweg-de Vries equations. Z. Angew. Math. Phys. 70(1), (2019), Paper No. 16, 28. https://doi.org/10.1007/ s00033-018-1060-4

21. Hausenblas, E.: Weak approximation for semilinear stochastic evolution equations. In: Stochastic analysis and related topics VIII. Vol. 53. Progr. Probab. Birkhäuser Basel, 2003, 111-128

22. Hausenblas, E.: Weak approximation of the stochastic wave equation. J. Comput. Appl. Math. 235(1), 33-58 (2010). https://doi.org/10.1016/j.cam.2010.03.026

23. Jacobe de Naurois, L., Jentzen, A., Welti, T.: Weak convergence rates for spatial spectral Galerkin approximations of semilinear stochastic wave equations with multiplicative noise. ArXiv e-prints (2015), 27 pages. arXiv:1508.05168 [math.PR]. Accepted in Appl. Math. Optim.

24. Jacobe de Naurois, L., Jentzen, A., Welti, T.: Lower Bounds for Weak Approximation Errors for Spatial Spectral Galerkin Approximations of Stochastic Wave Equations. In: Eberle, A., Grothaus, M., Hoh, W., Kassmann, M., Stannat, W., Trutnau, G. (eds.), Stochastic Partial Differential Equations and Related Fields. Springer International Publishing, Cham, 2018, 237-248. ISBN: 978-3-319-74929-7. https://doi.org/10.1007/978-3-319-74929-7_13

25. Jentzen, A., Kurniawan, R.: Weak convergence rates for Euler-type approximations of semilinear stochastic evolution equations with nonlinear diffusion coefficients. Found. Comput. Math. 21(2), 445-536 (2021). https://doi.org/10.1007/s10208-020-09448-x

26. Kloeden, P.E., Platen, E.: Numerical Solution of Stochastic Differential Equations. Vol. 23. Applications of Mathematics (New York). Springer-Verlag, Berlin, 1992, xxxvi+632. ISBN: 3-540-54062-8. https://doi.org/10.1007/978-3-662-12616-5

27. Kopec, M.: Quelques contributions à l'analyse numérique d'équations stochastiques. PhD thesis. École normale supérieure de Rennes - ENS Rennes, 2014

28. Kovács, M., Larsson, S., Lindgren, F.: Weak convergence of finite element approximations of linear stochastic evolution equations with additive noise. BIT 52(1), 85-108 (2012). https://doi.org/10.1007/ s10543-011-0344-2 
29. Kovács, M., Larsson, S., Lindgren, F.: Weak convergence of finite element approximations of linear stochastic evolution equations with additive noise II. Fully discrete schemes. BIT 53(2), 497-525 (2013). https://doi.org/10.1007/s10543-012-0405-1

30. Kovács, M., Larsson, S., Saedpanah, F.: Finite element approximation of the linear stochastic wave equation with additive noise. SIAM J. Numer. Anal. 48(2), 408-427 (2010). https://doi.org/10.1137/ 090772241

31. Kovács, M., Lindner, F., Schilling, R.L.: Weak convergence of finite element approximations of linear stochastic evolution equations with additive Lévy noise. SIAM/ASA J. Uncertain. Quantif. 3(1), 11591199 (2015). https://doi.org/10.1137/15M1009792

32. Kovács, M., Printems, J.: Weak convergence of a fully discrete approximation of a linear stochastic evolution equation with a positive-type memory term. J. Math. Anal. Appl. 413(2), 939-952 (2014). https://doi.org/10.1016/j.jmaa.2013.12.034

33. Lindgren, F.: On weak and strong convergence of numerical approximations of stochastic partial differential equations. PhD thesis. Chalmers University of Technology, 2012. ISBN: 978-91-7385787-1

34. Lindner, F., Schilling, R.L.: Weak order for the discretization of the stochastic heat equation driven by impulsive noise. Potential Anal. 38(2), 345-379 (2013). https://doi.org/10.1007/s11118-012-9276-y

35. Liu, W., Röckner M.: Stochastic Partial Differential Equations: An Introduction. Universitext. Springer, Cham, 2015, vi+266. ISBN: 978-3-319-22354-4. https://doi.org/10.1007/978-3-319-22354-4

36. Milstein, G. N.: Numerical Integration of Stochastic Differential Equations. Vol. 313. Mathematics and its Applications. Translated and revised from the 1988 Russian original. Kluwer Academic Publishers Group, Dordrecht, 1995, viii+169. ISBN: 0-7923-3213-X. https://doi.org/10.1007/978-94-015-84555

37. Müller-Gronbach, T., Ritter, K.: Lower bounds and nonuniform time discretization for approximation of stochastic heat equations. Found. Comput. Math. 7(2), 135-181 (2007). https://doi.org/10.1007/ s10208-005-0166-6

38. Müller-Gronbach, T., Ritter, K., Wagner, T.: Optimal pointwise approximation of infinitedimensional Ornstein-Uhlenbeck processes. Stoch. Dyn. 8(3), 519-541 (2008). https://doi.org/10. 1142/S0219493708002433

39. Quer-Sardanyons, L., Sanz-Solé, M.: Space semi-discretisations for a stochastic wave equation. Potential Anal. 24(4), 303-332 (2006). https://doi.org/10.1007/s11118-005-9002-0

40. Sell, G. R., You, Y.: Dynamics of Evolutionary Equations. Vol. 143. Applied Mathematical Sciences. Springer-Verlag, New York, 2002, xiv+670. ISBN: 0-387-98347-3. https://doi.org/10.1007/978-14757-5037-9

41. Shardlow, T.: Weak convergence of a numerical method for a stochastic heat equation. BIT 43(1), 179-193 (2003). https://doi.org/10.1023/A:1023661308243

42. Thomas, L. E.: Persistent energy flow for a stochastic wave equation model in nonequilibrium statistical mechanics. J. Math. Phys. 53(9), 095208 (2012). https://doi.org/10.1063/1.4728986

43. Walsh, J.B.: An introduction to stochastic partial differential equations. In: École d'Été de Probabilités de Saint-Flour, XIV-1984. Vol. 1180. Lecture Notes in Math. Springer, Berlin, 1986, 265-439. https:// doi.org/10.1007/BFb0074920

44. Walsh, J.B.: On numerical solutions of the stochastic wave equation. Illinois J. Math. 50(1-4), 9911018 (2006). https://doi.org/10.1215/ijm/1258059497

45. Wang, X.: An exponential integrator scheme for time discretization of nonlinear stochastic wave equation. J. Sci. Comput. 64(1), 234-263 (2015). https://doi.org/10.1007/s10915-014-9931-0

46. Wang, X.: Weak error estimates of the exponential Euler scheme for semi-linear SPDEs without Malliavin calculus. Discrete Contin. Dyn. Syst. 36(1), 481-497 (2016). https://doi.org/10.3934/dcds. 2016.36.481

47. Wang, X., Gan, S.: Weak convergence analysis of the linear implicit Euler method for semilinear stochastic partial differential equations with additive noise. J. Math. Anal. Appl. 398(1), 151-169 (2013). https://doi.org/10.1016/j.jmaa.2012.08.038

48. Wang, X., Gan, S., Tang, J.: Higher order strong approximations of semilinear stochastic wave equation with additive space-time white noise. SIAM J. Sci. Comput. 36(6), A2611-A2632 (2014). https://doi. org/10.1137/130937524

Publisher's Note Springer Nature remains neutral with regard to jurisdictional claims in published maps and institutional affiliations. 